\title{
Semantics of the second order lambda calculus $\uparrow$
}

\author{
BART JACOBS \\ Dep. Comp. Sc., Toernooiveld, 6525 ED Nijmegen, The Netherlands
}

Received 2 December 1989; revised 2 September 1990

\begin{abstract}
In the literature there are two main notions of model for the second order $\lambda$-calculus: one by Bruce, Meyer and Mitchell (the BMM-model, for short) in set-theoretical formulation and one category-theoretical by Seely. Here we generalise Seely's notion, using semifunctors and semi-adjunctions from Hayashi, and introduce $\lambda 2$-algebras, $\lambda \eta 2$-algebras, $\lambda 2$-models and $\lambda \eta 2$-models, similarly to the untyped $\lambda$-calculus. Non-extensional abstraction of both term and type variables is described by semi-adjunctions (essentially as in Martini's thesis). We show that also for second order sums, the $\beta$ - (and commutation!) conversions correspond to semi-functoriality and that the (additional) $\eta$-conversion corresponds to ordinary functoriality.

In the above framework various examples - well-known ones and variations - are described. Also, we determine the place of the BMM-models; an earlier version of the latter has been reported by Jacobs.
\end{abstract}

\section{Introduction}

The second order $\lambda$-calculus (denoted by $\lambda 2$ ), due to Girard (1972) and Reynolds (1974), is a typed $\lambda$-calculus with considerable computational strength, see e.g. Girard et al. (1989, chapters 11 and 15). In Bruce and Meyer (1984) a notion of model for this calculus was introduced which was formulated in set-theoretical terms; it was further developed in Bruce et al. (1990). In Seely (1987) another notion of model-a so-called PL-category - was introduced in category-theoretical terms. In Jacobs (1989), Seely's definition was adapted in a minor way - using the categorical non-extensional concepts from Hayashi (1985)such that these different notions of model could be translated back and forth. However a general theory of (non-extensional) semantics was still lacking; this will be the subject of the present paper. It will include a treatment of (non-extensional) second order sums and some relevant simple examples. Furthermore, the translations between BMM-models and categorical models from Jacobs (1989) are made functorial. The paper starts in sections 2-4 with a systematic exposition of material which is essentially already covered in the literature, see Hayashi (1985), Martini (1988), Coquand and Ehrhard (1987) and Pitts (1987).

It might be useful to mention two important reasons why category theory provides a suitable framework for the semantics of typed $\lambda$-calculi.

$\dagger$ Research partially supported by the 'Jumelage' project ST2J-0374-C of the European Communitee, and partially performed at the University of Pisa, Italy. 
(i) The notion of $\operatorname{Hom}_{\mathbf{C}}(A, B)$ - or $\mathbf{C}(A, B)$ - is very flexible; using all maps between two sets $A, B$ often leads to problems. To restrict oneself to certain functions - and define Hom $(A, B)$ accordingly - gives many possibilities, see subsection 7.1 where such a problem turns up for BMM-models.

(ii) In category theory one has suitable notions of indexing, e.g. in the form of indexed categories, fibrations or internal categories. Categorically, contexts form indices for the categories of types and terms derivable from those contexts. Such a 'double' indexing is harder to realise in set-theoretical language.

In this paper we therefore use the language of categories. A few more words about that: to someone not familiar with category theory, we mention that acquaintance with functors and natural transformations - which can be obtained easily - should be enough as a basis for this paper. To the more experienced category theorist we mention that we describe everything 'up-to-equality' (as is also done in Coquand and Ehrhard (1987) and Pitts (1987)). We do realise that this approach is a bit uncategorical, but we choose it nevertheless for practical reasons, see e.g. the formulation of the Beck-Chevalley condition in definition 3.1; it enables us to prove the substitution lemmas 4.1.1, 4.2.1 and 4.2.2 without too much effort. Anyway, the reader can always write for him/herself the canonical isomorphisms in the appropriate places.

In this paper we make extensive use of Hayashi's notions of semi-functor and semiadjunction to describe non-extensionality. This may cause some resistance, which is quite understandable, since people do not like to be restricted in their expressive power (probably for the same reason, intuitionistic mathematics never became very popular). Yet, we feel that there are at least four good reasons for doing so.

(1) The current computational paradigm is based on $\beta$-reduction and $\eta$-reduction is considered as secondary.

(2) There are non-extensional mathematical models for $\lambda 2$, see subsection 6.5.

(3) It provides useful insight to see exactly where one needs what.

(4) The 'semi-notions' have a remarkable generality. Although introduced by Hayashi in connection with the (first order) simple typed $\lambda$-calculus, the present paper shows that they can be used to describe second order products and sums as well. Of course, the applications do not stop here.

A consequence of the use of these semi-notions is that the categorical work becomes much more computational. This is due to the fact that one loses the possibility to do uniqueness diagram chases. Another consequence is that we have to start from scratch and reconsider a number of elementary matters, like substitution lemmas and the Frobenius reciprocity; this has to be done in a careful way.

\section{Preliminaries}

\subsection{Semi-functors, semi-adjunctions and semi-ccc's}

In Hayashi (1985) the notions of semi-functor and semi-adjunction are introduced in order to describe non-extensional concepts categorically. We recall the necessary elements. A semi-functor $F: \mathbf{C} \rightarrow \mathbf{D}$ is a 'functor' except that it needs not preserve identities. Another 
semi-functor $G: \mathbf{D} \rightarrow \mathbf{C}$ is a right semi-adjoint of $F$ - notation $F \dashv_{s} G$ - if there are collections of functions $\left\{\alpha_{X, Y}, \beta_{X, Y}\right\}_{X \in \mathbf{C}, Y \in \mathbf{D}}$ such that the big four squares in the following diagram commute.

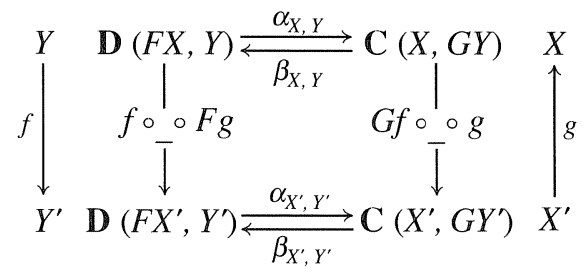

Lemma 2.1.1. Suppose $F \dashv_{s} G$ as described above, but with $F$ a (normal) functor; thenomitting indices - one has

(1) $\beta \circ \alpha=\mathrm{id}$,

i.e. $\mathbf{D}(F X, Y)$ is a retract of $\mathbf{C}(X, G Y)$.

(2) $\alpha(u \circ F v)=\alpha(u) \circ v \quad G u \circ \alpha(v)=\alpha(u \circ v)$.

(3) $\beta(u) \circ F v=\beta(u \circ v) \quad \beta(G u \circ v)=u \circ \beta(v)$.

Proof.

(1) $(\beta \circ \alpha)(u)=\operatorname{id} \circ \beta(\alpha(u)) \circ F$ id $=\beta(G$ id $\circ \alpha(u) \circ$ id $)=$ id $\circ u \circ F$ id $=u$.

(2) $\alpha(u \circ F v)=\alpha($ id $\circ u \circ F v)=G$ id $\circ \alpha(u) \circ v$

$=G$ id $\circ \alpha(u) \circ$ id $\circ v=\alpha($ id $\circ u \circ F$ id $) \circ v=\alpha(u) \circ v$.

$G u \circ \alpha(v)=G u \circ \alpha(v) \circ \mathrm{id}=\alpha(u \circ v \circ F \mathrm{id})=\alpha(u \circ v)$.

(3) Analogously.

An interesting remark, not mentioned in Hayashi (1985), is that in this case where $F$ is a normal functor, the pair $\left\langle G Y, \beta\left(\mathrm{id}_{G Y}\right): F G Y \rightarrow Y\right\rangle$ is weak universal from $F$ to $Y$ in the sense of MacLane (1971, X, 2). In comparison with this weak universality, the notion of semiadjunction incorporates a number of naturality conditions.

If both $F$ and $G$ are normal functors, the above diagram boils down to the usual one for an adjunction $F \dashv G$. Hence semi-adjunctions form a right generalisation.

A map of semi-adjunctions from $\langle F, G,\{\alpha, \beta\}\rangle: \mathbb{C} \rightarrow \mathbb{D}$ to $\left\langle F^{\prime}, G^{\prime},\left\{\alpha^{\prime}, \beta^{\prime}\right\}\right\rangle: \mathbb{C}^{\prime} \rightarrow \mathbb{D}^{\prime}$ consists of a pair of functors $\left\langle K: \mathbb{C} \rightarrow \mathbb{C}^{\prime}, L: \mathbb{D} \rightarrow \mathbf{D}^{\prime}\right\rangle$ such that

$$
\begin{aligned}
& L F=F^{\prime} K \quad \text { and } \quad G^{\prime} L=K G \text {, } \\
& K \alpha_{X, Y}=\alpha_{K X, L Y}^{\prime} L \text { and } L \beta_{X, Y}=\beta_{K X, L Y}^{\prime} K,
\end{aligned}
$$

as in MacLane (1971, IV, 7) for ordinary adjunctions.

A semi-ccc is defined as a category provided with the following three semi-adjunctions.

$$
\begin{gathered}
! \dashv_{s} t \\
\Delta\left({ }_{-}\right) \dashv_{s}(-) \times\left({ }_{-}\right) \\
(-) \times X \dashv_{s}()^{X} .
\end{gathered}
$$

Algebraically it can be described as follows.

(1) There is an object $t$ and for every object $X$ an arrow $!_{X}: X \rightarrow t$ such that $!_{Z} \circ f=!_{Y}$ for every $f: Y \rightarrow Z$. 
(2) For all objects $X, Y$ there is an object $X \times Y$ such that

(a) for all $f: Z \rightarrow X$ and $g: Z \rightarrow Y$ there is an arrow $\langle f, g\rangle: Z \rightarrow X \times Y$ satisfying

$$
\langle f, g\rangle \circ h=\langle f \circ h, g \circ h\rangle \text {. }
$$

(b) there are morphisms $\pi: X \times Y \rightarrow X$ and $\pi^{\prime}: X \times Y \rightarrow Y$ with

$$
\begin{aligned}
\pi \circ\langle f, g\rangle & =f, \\
\pi^{\prime} \circ\langle f, g\rangle & =g .
\end{aligned}
$$

(3) For all objects $X, Y$ there is an object $Y^{X}$ such that

(a) for every $h: Z \times X \rightarrow Y$ there is an arrow $\Lambda(h): Z \rightarrow Y^{X}$;

(b) there is an arrow $e v: Y^{X} \times X \rightarrow Y$ with

$$
\begin{aligned}
e v \circ\langle\Lambda(h) \circ f, g\rangle & =h \circ\langle f, g\rangle \\
\Lambda\left(h \circ\left\langle f \circ \pi, \pi^{\prime}\right\rangle\right) & =\Lambda(h) \circ f \\
e v \circ\left\langle\pi, \pi^{\prime}\right\rangle & =e v .
\end{aligned}
$$

Hence a semi-ccc as described above is a (normal) ccc iff

(1) $!_{t}=\mathrm{id}_{t}$.

(2) $\left\langle\pi, \pi^{\prime}\right\rangle=$ id. (surjectivity of pairing)

(3) $\Lambda(e v)=\mathrm{id} . \quad(\eta)$

In Hayashi (1985) one can find that the Karoubi envelope of a semi-ccc yields a ccc, which can be described by a universal property.

These semi-notions are easier to understand in a negative way: a semi-functor is a functor except that ...; a semi-ccc is a ccc except that...; for this reason we speak about 'non-extensionality' instead of e.g. 'intensionality'.

In a semi-ccc one can define finite products as usual; the details are as follows. For $n \geqslant 2$, put

$$
X_{0} \times \ldots \times X_{n}=\left(X_{0} \times \ldots \times X_{n-1}\right) \times X_{n}
$$

and for $0 \leqslant i \leqslant n$, define

$$
\pi_{i}^{n}: X_{0} \times \ldots \times X_{n} \rightarrow X_{i}
$$

by

$$
\pi_{0}^{0}=\mathrm{id}_{X_{0}} \quad \pi_{i}^{n+1}= \begin{cases}\pi^{\prime} & \text { if } i=n+1 \\ \pi_{i}^{n} \circ \pi & \text { else. }\end{cases}
$$

Furthermore, for $f_{0}: Y \rightarrow X_{0}, \ldots, f_{n}: Y \rightarrow X_{n}$, put

$$
\left\langle f_{0}\right\rangle=f_{0} \quad\left\langle f_{0}, \ldots, f_{i+1}\right\rangle=\left\langle\left\langle f_{0}, \ldots, f_{i}\right\rangle, f_{i+1}\right\rangle .
$$

Then one has $\pi_{i}^{n} \circ\left\langle f_{0}, \ldots, f_{n}\right\rangle=f_{i}$ and $\left\langle f_{0}, \ldots, f_{n}\right\rangle \circ g=\left\langle f_{0} \circ g, \ldots, f_{n} \circ g\right\rangle$. Only in a ccc one has $\left\langle\pi_{0}^{0}, \ldots, \pi_{n}^{n}\right\rangle=\mathrm{id}_{X_{0} \times \ldots \times X_{n}}$. 


\subsection{First order semantical notions}

Before we can give model definitions, some technicalities have to be established. Remember that an object $X$-in a category $\mathbf{C}$ with (semi-) terminal object $t$ - has enough points if for all $f, g: X \rightarrow Y$ with $\forall x: t \rightarrow X$. $f \circ x=g \circ x$ one has $f=g$. In Goldblatt (1984, p. 116) this property is called the extensionality principle for arrows. If all objects of $\mathbf{C}$ have enough points, C is called well-pointed. In that case, MacLane (1971, p. 123) speaks of $t$ as a generator.

Definition 2.2.1. Let $\mathbf{C}$ be a semi-ccc.

(1) ('application in C') For $f: t \rightarrow Y^{X}$ and $x: t \rightarrow X$, put

$$
f \cdot x=e v \circ\langle f, x\rangle: t \rightarrow Y \text {. }
$$

(2) The name $\lceil f\rceil$ of an arrow $f: X \rightarrow Y$ is defined by

$$
\lceil f\rceil=\Lambda\left(f \circ \pi^{\prime}\right): t \rightarrow Y^{X} .
$$

Then one has $[f\rceil \cdot x=f \circ x$. As a special case we write $1=\lceil\Lambda(e v)]: t \rightarrow\left(Y^{X}\right)^{\left(Y^{X}\right)}$.

(3) We say that $\mathbf{C}$ satisfies the Meyer-Scott axiom if for all $f, g: t \rightarrow Y^{X}$,

$$
\forall x: t \rightarrow X . f \cdot x=g \cdot x \Rightarrow 1 \cdot f=1 \cdot g .
$$

What we call the Meyer-Scott axiom is a typed version of the axiom with the same name used in the semantics of the untyped $\lambda$-calculus, see Barendregt (1984, 5.2.7.(ii)). This Meyer-Scott axiom is in a non-extensional context a little bit stronger than the condition of well-pointedness and therefore more suitable.

Proposition 2.2.2. Let $\mathbf{C}$ be a semi-ccc.

(1) $\mathbf{C}$ satisfies the Meyer-Scott axiom $\Rightarrow \mathbf{C}$ is well-pointed.

(2) $\mathrm{C}$ is well-pointed and has a normal terminal object $\Rightarrow \mathbf{C}$ satisfies the Meyer-Scott axiom.

Proof. (1) First we notice two facts.

Given an arrow $g: t \rightarrow Y^{X}$, we can write $\lfloor g\rfloor=e v \circ\left\langle g \circ !_{X}, \operatorname{id}_{X}\right\rangle: X \rightarrow Y$. Then

$$
\lfloor[f\rceil]=e v \circ\left\langle\Lambda\left(f \circ \pi^{\prime}\right) \circ !_{X}, \mathrm{id}_{X}\right\rangle=f \circ \pi^{\prime} \circ\left\langle!_{X}, \mathrm{id}_{X}\right\rangle=f .
$$

Furthermore,

$$
\begin{aligned}
1 \cdot\lceil f\rceil & =\Lambda(e v) \circ\lceil f\rceil \\
& =\Lambda\left(e v \circ\left\langle\Lambda\left(f \circ \pi^{\prime}\right) \circ \pi, \pi^{\prime}\right\rangle\right) \\
& =\Lambda\left(f \circ \pi^{\prime} \circ\left\langle\pi, \pi^{\prime}\right\rangle\right) \\
& =\lceil f\rceil .
\end{aligned}
$$

So in order to provide the first statement, suppose that $f, g: X \rightarrow Y$;

$$
\begin{aligned}
\forall x: t \rightarrow X . f \circ x & =g \circ x \\
& \Rightarrow \forall x: t \rightarrow X .[f\rceil \cdot x=\lceil g] \cdot x \\
& \Rightarrow 1 \cdot[f]=1 \cdot[g], \quad \text { by the Meyer-Scott axiom } \\
& \Rightarrow f=g, \quad \text { by the above facts. }
\end{aligned}
$$


(2) Suppose now that $f, g: t \rightarrow Y^{X}$;

$$
\begin{aligned}
\forall x: t \rightarrow X . f \cdot x= & g \cdot x \\
\Rightarrow & \forall y: t \rightarrow t \times X . f \cdot\left(\pi^{\prime} \circ y\right)=g \cdot\left(\pi^{\prime} \circ y\right) \\
\Rightarrow & \forall y: t \rightarrow t \times X . e v \circ\left\langle f, \pi^{\prime} \circ y\right\rangle=e v \circ\left\langle g, \pi^{\prime} \circ y\right\rangle \\
\Rightarrow & \forall y: t \rightarrow t \times X . e v \circ\left\langle f \circ \pi, \pi^{\prime}\right\rangle \circ y=e v \circ\left\langle f \circ \pi, \pi^{\prime}\right\rangle \circ y, \\
& \text { since } \pi \circ y=\mathrm{id}_{t} \text { because the terminal object is normal. } \\
\Rightarrow & e v \circ\left\langle f \circ \pi, \pi^{\prime}\right\rangle=e v \circ\left\langle g \circ \pi, \pi^{\prime}\right\rangle, \\
& \text { since C is well-pointed. } \\
\Rightarrow & 1 \cdot f=\Lambda\left(e v \circ\left\langle f \circ \pi, \pi^{\prime}\right\rangle\right)=\Lambda\left(e v \circ\left\langle g \circ \pi, \pi^{\prime}\right\rangle\right)=1 \cdot g .
\end{aligned}
$$

Combining these results with the connection established in Hayashi (1985, section 3) between semi-ccc's and the simple typed $\lambda$-calculus (denoted here by $\lambda 1$ ), we present the following notions of first order models.

Definition 2.2.3. A category $\mathbb{C}$ will be called

(1) a $\lambda$ 1-algebra if $\mathbf{C}$ is a semi-ccc;

(2) a $\lambda 1$-model if $\mathbf{C}$ is a semi-cce satisfying the Meyer-Scott axiom;

(3) a $\lambda \eta 1$-algebra if $\mathbf{C}$ is a ccc;

(4) a $\lambda \eta 1$-model if $\mathbf{C}$ is a ccc satisfying the Meyer-Scott axiom.

Hence a $\lambda \eta 1$-model is just a well-pointed ccc. Such a category may be called extensional since it satisfies $\forall x \cdot f \cdot x=g \cdot x \Rightarrow f=g$.

\subsection{Syntax of the second order $\lambda$-calculus}

The syntax of the second order $\lambda$-calculus $\lambda 2$ is introduced in two steps.

Types. The statement $\Gamma \vdash \sigma: *$, expressing that $\sigma$ is a type in context $\Gamma$ is defined as follows. Let $\Gamma=\left\{\gamma_{1}: *, \ldots, \gamma_{n}: *\right\}$ be a set of type variables; $\Gamma, \gamma: *$ denotes $\Gamma \cup\{\gamma: *\}$, where it is supposed that $\gamma: * \notin \Gamma$. The deduction rules for types are

$$
\begin{aligned}
\gamma: * \in \Gamma & \Rightarrow \Gamma \vdash \gamma: * \\
\Gamma \vdash \sigma: *, \Gamma \vdash \tau: * & \Rightarrow \Gamma \vdash \sigma \rightarrow \tau: * \\
\Gamma, \gamma: * \vdash \sigma: * & \Rightarrow \Gamma \vdash \forall \gamma: * . \sigma: * .
\end{aligned}
$$

Terms. The statement $\Gamma+\Theta \vdash M: \sigma$, denoting that the term $M$ has type $\sigma$ in the type context $\Gamma$ and the term context $\Theta$ is formed as follows. Let $\Theta=\left\{x_{1}: \sigma_{1}, \ldots, x_{m}: \sigma_{m}\right\}$ be a set of statements about term variables such that $\Gamma \vdash \sigma_{i}: *$; the context $\Theta, y: \tau$ is $\Theta \cup\{y: \tau\}$, 
where $y: \tau \notin \Theta$. We write $\gamma \in F V(\Theta)$ iff there is an $i \leqslant n$ with $\gamma \in F V\left(\sigma_{i}\right)$. The deduction rules for terms are

$$
\begin{aligned}
& x: \tau \in \Theta \Rightarrow \Gamma+\Theta \vdash x: \tau \\
& \Gamma+\Theta \vdash M: \sigma \rightarrow \tau, \Gamma+\Theta \vdash N: \sigma \Rightarrow \Gamma+\Theta \vdash M N: \tau \\
& \Gamma+\Theta, x: \sigma \vdash M: \tau \Rightarrow \Gamma+\Theta \vdash \lambda x: \sigma . M: \sigma \rightarrow \tau \\
& \Gamma+\Theta \vdash M: \forall \gamma: * . \sigma, \Gamma \vdash \tau: * \Rightarrow \Gamma+\Theta \vdash M \tau: \sigma[\gamma:=\tau] \\
& \Gamma, \gamma: *+\Theta \vdash M: \sigma \quad \text { and } \quad \gamma \notin F V(\Theta) \Rightarrow \Gamma+\Theta \vdash \lambda \gamma: * . M: \forall \gamma: * . \sigma \text {. }
\end{aligned}
$$

The $\beta$-conversions are

$$
(\lambda x: \sigma . M) N=M[x:=N] \text { and }(\lambda \gamma: * . M) \tau=M[\gamma:=\tau] .
$$

Especially, we mention the $\xi$-rules

$$
\begin{array}{r}
\Gamma+\Theta, x: \sigma \vdash M=N \Rightarrow \Gamma+\Theta \vdash \lambda x: \sigma . M=\lambda x: \sigma . N \\
\Gamma, \gamma: *+\Theta \vdash M=N \quad \text { and } \quad \gamma \notin F V(\Theta) \Rightarrow \Gamma+\Theta \vdash \lambda \gamma: * . M=\lambda \gamma: * . N .
\end{array}
$$

The calculus $\lambda \eta 2$ has the extra $\eta$-conversions

$$
\lambda x: \sigma \cdot M x=M \quad \text { and } \quad \lambda \gamma: * . M \gamma=M,
$$

if $x, \gamma$ do not occur in $M$.

The above separation of contexts is possible because there is no type dependency in the calculus $\lambda 2$ (i.e. term variables cannot occur in types). This makes the semantics relatively simple.

\section{Second order semantical notions}

As in subsection 2.2 for first order semantics (and as for the untyped $\lambda$-calculus, see Barendregt, 1984, ch. 5), second order semantics is developed in two directions. The basic notion on which we make variations are the (second order version of the) PL-categories from Seely (1987); in this presentation they will be called $\lambda \eta 2$-algebras. We start from the more general notion of a $\lambda 2$-algebra, which is essentially as in Martini (1988, 2, 5.9).

Definition 3.1. A $\lambda 2$-algebra $H$ consists of the following data.

(1) A base category $\mathbf{B}$ with finite products (including a terminal object $t$ ) and with a distinguished object $\Omega$.

(2) A functor $H: \mathbf{B}^{\text {op }} \rightarrow$ Cat satisfying

(a) for $A \in \mathbf{B}$, there is an isomorphism $\phi_{A}: \mathbf{B}(A, \Omega) \cong \operatorname{Obj}(H A)$, natural in the following way: for $u: B \rightarrow A$ in $\mathbf{B}$, the functor $u^{*}=H u: H A \rightarrow H B$ acts on objects $X \in H A$ as

$$
u^{*}(X)=\phi_{B}\left(\phi_{A}^{-1}(X) \circ u\right)
$$

(b) the fibre categories $H A$ are semi-ccc's; the functors $u^{*}$ preserve the semi-ccc structure. 
(3) For $A \in \mathbf{B}$, there is a semi-functor $\Pi_{A}: H(A \times \Omega) \rightarrow H A$ which is a right semi-adjoint of $\pi_{A, \Omega}^{*}$. Moreover, a Beck-Chevalley condition is satisfied: if $u: B \rightarrow A$ in $\mathbf{B}$, then $\left\langle u^{*},\left(u \times \mathrm{id}_{\Omega}\right)^{*}\right\rangle$ is a map from the semi-adjunction $\pi_{A, \Omega}^{*} \dashv_{s} \Pi_{A}$ to $\pi_{B, \Omega}^{*} \dashv_{s} \Pi_{B}$.

This formulation of the Beck-Chevalley condition expresses (cf. subsection 2.1) that if

$$
H(A \times \Omega)\left(\pi_{A, \Omega}^{*}(X), Y\right) \underset{\beta(A)_{X, Y}}{\stackrel{\alpha(A)_{X, Y}}{\rightleftarrows}} H(A)\left(X, \Pi_{A}(Y)\right)
$$

describes the semi-adjunction $\pi_{A, \Omega}^{*} \dashv_{s} \Pi_{A}$, then for $u: B \rightarrow A$ in $\mathbf{B}$ one has

$$
\begin{aligned}
\prod_{B} \circ\left(u \times \mathrm{id}_{\Omega}\right)^{*} & =u^{*} \circ \prod_{A} \\
u^{*} \alpha(A)_{X, Y} & =\alpha(B)_{u^{*}(X),\left(u \times \mathrm{id}_{\Omega}\right)^{*}(Y)}\left(u \times \mathrm{id}_{\Omega}\right)^{*} \\
\left(u \times \mathrm{id}_{\Omega}\right)^{*} \beta(A)_{X, Y} & =\beta(B)_{u^{*}(X),{ }^{*}\left(u \times \mathrm{id}_{\Omega}\right)^{*}(Y)} u^{*} .
\end{aligned}
$$

Remarks 3.1.1. (1) As mentioned above, we work with the second order version of Seely's PL-categories, in the original (higher order) definition (Seely 1987, 2.1) one additionally has exponents in the basis and more general product functors $\Pi_{A, C}: H(A \times C) \rightarrow H A$. Of course one can generalise these to the non-extensional case as well. In that case the base category must have semi-products instead of the ordinary ones that we require above. We use these products only to arrange type variables; they are not 'semi' for pragmatical reasons.

(2) From Seely $(1987,2.2(2))$. There is a map $\psi_{A}: \mathbf{B}(A, \Omega \times \Omega) \dot{\rightarrow}(A, \Omega)$

$$
u \mapsto \phi_{A}^{-1}\left(\phi_{A}(\pi \circ u)^{\phi_{A}\left(\pi^{\prime} \circ u\right)}\right),
$$

which is natural in $A$. The Yoneda lemma then induces a map $\leadsto=\psi_{\Omega \times \Omega}($ id $): \Omega \times \Omega \rightarrow \Omega$ in B such that $\psi(u)=\rightsquigarrow \circ u$. This same arrow turns up for BMM-models, see section 7 .

(3) In case a $\lambda 2$-algebra $H$ has normal adjunctions $\pi_{A, \Omega}^{*}-\dashv \Pi_{A}$, the Beck-Chevalley condition can be expressed in the usual way: for $u: B \rightarrow A$ in $\mathbf{B}$, the canonical natural transformation $\alpha(B)\left(\left(u \times \mathrm{id}_{\Omega}\right)^{*} \beta(A)(\mathrm{id})\right): u^{*} \Pi_{A} \rightarrow \Pi_{B}\left(u \times \mathrm{id}_{\Omega}\right)^{*}$ should be the identity. (The identity and not an isomorphism, since we work up-to-equality.)

(4) The domain theoretic model from Coquand et al. (1989) fits nicely in the above hyperdoctrine framework (in fact, one gets a $\lambda \eta 2$-model, see definition 3.2 below): the objects of the fibres become continuous functors $F:\left(D^{E P}\right)^{n} \rightarrow D^{E P}$ and morphisms $s: F \rightarrow G$ will be continuous sections of the fibration $\Sigma(F \Rightarrow G) \rightarrow\left(D^{E P}\right)^{n}$. This remark is intended to show that - the other way round-definition 3.1 can also be seen in the framework of fibrations and continuous sections. Therefore, we write $|A|$, where $A$ is an object in a category with (semi-) terminal $t$, for the discrete category with arrows $a: t \rightarrow A$ as objects. An object $X \in H A$ as in definition 3.1 yields a (discrete) indexed category $\hat{X}:|A| \rightarrow$ Cat by $a \mapsto\left|a^{*}(X)\right|$. Applying the Grothendieck construction to $\hat{X}$ yields a fibration $\Sigma \hat{X} \rightarrow|A|$, a section of which consists of a family $\left\{s_{a}\right\}_{a \in|A|}$ with $s_{a} \in\left|a^{*}(X)\right|$. An arrow $f: X \rightarrow Y$ in $H A$ then gives rise to a section $\left\{a^{*}([f\rceil)\right\}_{a \in|A|}$ of the fibration $\Sigma\left(Y^{X}\right) \rightarrow|A|$. The fact that Coquand et al. use continuous functors and sections comes from the context of Scott domains in which they work. 
Definition 3.1.2. We take as an arrow between two $\lambda 2$-algebras $H: \mathbf{B}^{\text {op }} \rightarrow$ Cat and $H^{\prime}: \mathbf{B}^{\prime o p} \rightarrow$ Cat, a pair $\langle R, S\rangle$ where

$$
R: \mathbf{B} \rightarrow \mathbf{B}^{\prime}
$$

is a functor that preserves the cc-structure and $\Omega$

$$
S: H \rightarrow H^{\prime} R
$$

is a natural transformation such that its component-functors $S_{a}$ preserve the semi-ccc structure and commute with the $\phi$ 's; moreover, the pair $\left\langle S_{A}, S_{A \times \Omega}\right\rangle$ is a map from $\pi_{A, \Omega}^{*} \dashv_{s} \Pi_{A}$ to $\pi_{R A, \Omega^{\prime}}^{* \prime} \dashv_{s} \Pi_{A}^{\prime}$. In detail, one has

$$
S_{A} \phi_{A}=\phi_{R A}^{\prime} R
$$

and with respect to the semi-adjunctions (omitting subscripts),

$$
\begin{aligned}
\prod_{R A}^{\prime} \circ S_{A \times \Omega} & =S_{A} \circ \prod_{A} \\
S_{A} \alpha(A) & =\alpha(R A)^{\prime} S_{A \times \Omega} \\
S_{A \times \Omega} \beta(A) & =\beta(R A)^{\prime} S_{A} .
\end{aligned}
$$

In this way a category $\lambda 2$-ALG is obtained. There is an obvious forgetful functor $\lambda 2$-ALG $\rightarrow$ Ind, the category of indexed categories.

Definition 3.2. Let $H$ be a $\lambda 2$-algebra as described above; $H$ will be called:

(1) A $\lambda 2$-model if the fibre category $H t$ is a $\lambda 1$-model and if the functor $H$ preserves enough points of $\Omega$, i.e. for arrows $f, g: X \rightarrow Y$ in $H \Omega$ with $\forall u: t \rightarrow \Omega . u^{*}(f)=u^{*}(g)$ one has $f=g$.

(2) A $\lambda \eta 2$-algebra if the fibres $H A$ are ccc's and the adjunctions $\pi_{A, \Omega}^{*} \dashv \Pi_{A}$ are ordinary ones.

(3) an $\lambda \eta 2$-model if $H$ is both a $\lambda 2$-model and a $\lambda \eta 2$-algebra.

Thus we have full subcategories $\lambda 2$-MOD, $\lambda \eta 2-$ ALG and $\lambda \eta 2-$ MOD of $\lambda 2$-ALG.

Finally one more subcategory is needed in order to deal with BMM-models in section 7.

Definition 3.3. A $\lambda(\eta)$ 2-algebra (or model) $H: \mathbf{B}^{\mathrm{op}} \rightarrow$ Cat will be called small if

(a) the collection $\mathbf{B}(t, \Omega)$ is a (small) set;

(b) the collections $H t\left(t^{\prime}, X\right)$ are (small) sets for objects $X$ of $H t$ (where $t^{\prime}$ is semi-terminal object in the fibre category $H t$ );

(c) every product $\Omega \times \ldots \times \Omega \in \mathbf{B}$ has enough points.

In this way we form categories $\lambda(\eta) 2-\mathbf{A L G}_{\mathrm{s}}$ and $\lambda(\eta) 2-\mathbf{M O D}_{\mathrm{s}}$ (with subscript ' $\mathrm{s}$ ').

\section{Interpretation}

In this section we describe the interpretation of the types and terms of the calculus $\lambda 2$ in a fixed $\lambda 2$-algebra $H$ as determined in definition 3.1 above. The approach is as in Koymans (1982) for the untyped $\lambda$-calculus. 
Types 4.1. Suppose a type statement $\Gamma \vdash \sigma: *$ is given for $\Gamma=\left\{\gamma_{1}: *, \ldots, \gamma_{n}: *\right\}$; implicitly it is supposed that contexts have a certain ordering. We put $\llbracket \Gamma \rrbracket=t \times \Omega \times \ldots \times \Omega(n$ times $\Omega)$ and define $\llbracket \sigma \rrbracket_{\Gamma}: \llbracket \Gamma \rrbracket \rightarrow \Omega$ in $\mathbf{B}$ as follows.

$$
\begin{aligned}
{\left[\gamma_{i} \rrbracket_{\Gamma}\right.} & =\pi_{i}^{n} \\
\llbracket \sigma \rightarrow \tau \rrbracket_{\Gamma} & =\phi_{[\Gamma]}^{-1}\left(\phi_{\llbracket \Gamma]}\left(\llbracket \tau \rrbracket_{\Gamma}\right)^{\phi_{\llbracket \Gamma]}\left(\left[\sigma \rrbracket_{\Gamma}\right)\right.}\right) \\
\llbracket \forall \gamma: * . \sigma \rrbracket_{\Gamma} & =\phi_{[\Gamma]}\left(\Pi_{[\Gamma]}\left(\phi_{[\Gamma, \gamma: *]}\left(\llbracket \sigma \rrbracket_{\Gamma, \gamma: *}\right)\right)\right) .
\end{aligned}
$$

Lemma 4.1.1. ('Type substitution'.) Let $\Gamma=\left\{\gamma_{1}: *, \ldots, \gamma_{n}: *\right\} \vdash \sigma, \tau: *$.

(1) If $\gamma_{i} \notin F V(\sigma)$, then $\llbracket \sigma \rrbracket_{\Gamma}=\llbracket \sigma \rrbracket_{\Gamma-\gamma_{i}: *} \circ\left\langle\pi_{0}^{n}, \ldots, \pi_{i-1}^{n}, \pi_{i+1}^{n}, \ldots, \pi_{n}^{n}\right\rangle$.

(2) $\llbracket \sigma\left[\gamma_{i}:=\tau\right] \rrbracket_{\Gamma}=\llbracket \sigma \rrbracket_{\Gamma} \circ\left\langle\pi_{0}^{n}, \ldots, \pi_{i-1}^{n}, \llbracket \tau \rrbracket_{\Gamma}, \pi_{i+1}^{n}, \ldots, \pi_{n}^{n}\right\rangle$.

(3) If $\gamma_{n} \notin F V(\tau)$, then $\llbracket \sigma\left[\gamma_{n}:=\tau\right] \rrbracket_{\Gamma-\gamma_{n}: *}=\llbracket \sigma \rrbracket_{\Gamma} \circ\left\langle\mathrm{id}, \llbracket \tau \rrbracket_{\Gamma-\gamma_{n}: *}\right\rangle$.

Proof. (1) $+(2)$ are done by induction on the derivation of $\sigma$, using Beck-Chevalley in case $\sigma \equiv \forall \gamma: * . \sigma_{1}$. For (3), we write $a=\llbracket \tau \rrbracket_{\Gamma-\gamma_{n}: *}: t \times \Omega \times \ldots \times \Omega \rightarrow \Omega(n-1$ times). From (i) we know that $\llbracket \tau \rrbracket_{\Gamma}=a \circ \pi$ and so that $a=\llbracket \tau \rrbracket_{\Gamma} \circ\langle$ id, $a\rangle$. Hence

$$
\begin{aligned}
\llbracket \sigma \rrbracket_{\Gamma} \circ\langle\mathrm{id}, a\rangle & =\llbracket \sigma \rrbracket_{\Gamma} \circ\left\langle\pi, \llbracket \tau \rrbracket_{\Gamma}\right\rangle \circ\langle\mathrm{id}, a\rangle \\
& =\llbracket \sigma\left[\gamma_{n}:=\tau\right] \rrbracket_{\Gamma} \circ\langle\mathrm{id}, a\rangle \quad \text { by }(2) \\
& =\llbracket \sigma\left[\gamma_{n}:=\tau\right] \rrbracket_{\Gamma-\gamma_{n}: *} \circ \pi \circ\langle\mathrm{id}, a\rangle \quad \text { by }(1) \\
& =\llbracket \sigma\left[\gamma_{n}:=\tau\right] \rrbracket_{\Gamma-\gamma_{n}: *} .
\end{aligned}
$$

Terms 4.2. Suppose we have $\Gamma+\Theta \vdash M: \sigma$ with $\Gamma=\left\{\gamma_{1}: *, \ldots, \gamma_{n}: *\right\}$ as before and $\Theta=\left\{x_{1}: \tau_{1}, \ldots, x_{m}: \tau_{m}\right\}$. We write $\llbracket \Theta \rrbracket_{\Gamma}=t \times \phi_{[\Gamma]}\left(\llbracket \tau_{1} \rrbracket_{\Gamma}\right) \times \ldots \times \phi_{[\Gamma]}\left(\llbracket \tau_{m} \rrbracket_{\Gamma}\right)$, which is an object of $H \llbracket \Gamma \rrbracket$.

An arrow $\llbracket M \rrbracket_{\Gamma+\Theta}: \llbracket \Theta \rrbracket_{\Gamma} \rightarrow \phi_{[\Gamma]}\left(\llbracket \sigma \rrbracket_{\Gamma}\right)$ in $H \llbracket \Gamma \rrbracket$ will be defined as follows.

$$
\begin{aligned}
\llbracket x_{j} \rrbracket_{\Gamma+\Theta} & =\pi_{j}^{n} \\
\llbracket \lambda y: \mu . M \rrbracket_{\Gamma+\Theta} & =\Lambda\left(\llbracket M \rrbracket_{\Gamma+\Theta, y ; \mu}\right) \\
\llbracket M N \rrbracket_{\Gamma+\Theta} & =e v \circ\left\langle\llbracket M \rrbracket_{\Gamma+\Theta}, \llbracket N \rrbracket_{\Gamma+\Theta}\right\rangle \\
\llbracket \lambda \gamma: * . M \rrbracket_{\Gamma+\Theta} & =\alpha(\llbracket \Gamma \rrbracket)\left(\llbracket M \rrbracket_{\Gamma, \gamma: *+\Theta}\right) . \\
\llbracket M \mu \rrbracket_{\Gamma+\Theta} & =\left\langle\mathrm{id}, \llbracket \mu \rrbracket_{\Gamma}\right\rangle^{*}(\beta(\llbracket \Gamma \rrbracket)(\mathrm{id})) \circ \llbracket M \rrbracket_{\Gamma+\Theta} .
\end{aligned}
$$

(The subscripts belonging to $\alpha(\llbracket \Gamma \rrbracket)$ and $\beta(\llbracket \Gamma \rrbracket)$ have been omitted in order to increase the readability, see below definition 3.1 for the details.)

In order to prove a soundness theorem, the following technical results are needed. The first point of lemma 4.2.2 is crucial in a non-extensional setting; the rest is more or less standard.

Lemma 4.2.1. ('Type in term substitution'.) Let $\Gamma+\Theta \vdash M: \sigma$ for $\Gamma=\left\{\gamma_{1}: *, \ldots, \gamma_{n}: *\right\}$.

(1) If $\gamma_{i} \notin \Theta$ and $\gamma_{i} \notin F V(M)$, then

$$
\llbracket M \rrbracket_{\Gamma+\Theta}=\left\langle\pi_{0}^{n}, \ldots, \pi_{i-1}^{n}, \pi_{i+1}^{n}, \ldots, \pi_{n}^{n}\right\rangle *\left(\llbracket M \rrbracket_{\Gamma-\gamma_{i}: *+\Theta}\right) .
$$


(2) If $\Gamma \vdash \tau: *$, then

$$
\llbracket M\left[\gamma_{i}:=\tau\right] \rrbracket_{\Gamma+\Theta}=\left\langle\pi_{0}^{n}, \ldots, \pi_{i-1}^{n}, \llbracket \tau \rrbracket_{\Gamma}, \pi_{i+1}^{n}, \ldots, \pi_{n}^{n}\right\rangle^{*}\left(\llbracket M \rrbracket_{\Gamma+\Theta}\right) .
$$

If $\gamma_{n} \notin F V(\tau)$, then

$$
\llbracket M\left[\gamma_{n}:=\tau\right] \rrbracket_{\Gamma-\gamma_{n}: *}=\left\langle\mathrm{id}, \llbracket \tau \rrbracket_{\Gamma-\gamma_{n}: *}\right\rangle *\left(\llbracket M \rrbracket_{\Gamma+\Theta}\right) .
$$

(3) $\llbracket(\lambda \gamma: * . P) \tau \rrbracket_{\Gamma+\Theta}=\llbracket P[\gamma:=\tau] \rrbracket_{\Gamma+\Theta}$.

Proof. (1) $+(2)$ are done by induction on the derivation of $M$, using Beck-Chevalley and lemma 4.1.1. As for (3),

$$
\begin{aligned}
\llbracket(\lambda \gamma: * . P) \tau \rrbracket_{\Gamma+\Theta} & =\left\langle\mathrm{id}, \llbracket \tau \rrbracket_{\Gamma}\right\rangle^{*}(\beta(\llbracket \Gamma \rrbracket)(\mathrm{id})) \circ \alpha(\llbracket \Gamma \rrbracket)\left(\llbracket P \rrbracket_{\Gamma, \gamma ; *+\Theta}\right) \\
& =\left\langle\mathrm{id}, \llbracket \tau \rrbracket_{\Gamma}\right\rangle^{*}\left\{\beta(\llbracket \Gamma \rrbracket)(\mathrm{id}) \circ \pi_{\llbracket \Gamma, \Omega}^{*} \alpha(\llbracket \Gamma \rrbracket)\left(\llbracket P \rrbracket_{\Gamma, \gamma: *+\Theta}\right)\right\} \\
& =\left\langle\mathrm{id}, \llbracket \tau \rrbracket_{\Gamma}\right\rangle^{*}\left\{\beta(\llbracket \Gamma \rrbracket) \circ \alpha(\llbracket \Gamma \rrbracket)\left(\llbracket P \rrbracket_{\Gamma, \gamma: *+\Theta}\right)\right\} \\
& =\left\langle\mathrm{id}, \llbracket \tau \rrbracket_{\Gamma}\right\rangle^{*}\left\{\llbracket P \rrbracket_{\Gamma, \gamma: *+\Theta}\right\}, \quad \text { see lemma } 2.1 .1 \\
& =\llbracket P[\gamma:=\tau] \rrbracket_{\Gamma+\Theta}, \quad \text { by }(2) .
\end{aligned}
$$

Lemma 4.2.2. ('Term in term substitution'.) Let $\Gamma+\Theta \vdash M: \sigma$ for $\Theta=\left\{x_{1}: \tau_{1}, \ldots, x_{m}: \tau_{m}\right\}$.

(1) $\llbracket M \rrbracket_{\Gamma+\Theta}=\llbracket M \rrbracket_{\Gamma+\Theta} \circ\left\langle\pi_{0}^{m}, \ldots, \pi_{m}^{m}\right\rangle$.

(2) If $x_{i} \notin F V(M)$, then

$$
\llbracket M \rrbracket_{\Gamma+\Theta}=\llbracket M \rrbracket_{\Gamma+\Theta-x_{i}: \tau_{i}} \circ\left\langle\pi_{0}^{m}, \ldots, \pi_{i-1}^{m}, \pi_{i+1}^{m}, \ldots, \pi_{m}^{m}\right\rangle .
$$

If $i=m$, this becomes

$$
\llbracket M \rrbracket_{\Gamma+\Theta}=\llbracket M \rrbracket_{\Gamma+\Theta-x_{m}: \tau_{m}} \circ \pi
$$

(3) If $\Gamma+\Theta \vdash N: \tau_{i}$, then

$$
\llbracket M\left[x_{i}:=N\right] \rrbracket_{\Gamma+\Theta}=\llbracket M \rrbracket_{\Gamma+\Theta} \circ\left\langle\pi_{0}^{m}, \ldots, \pi_{i-1}^{m}, \llbracket N \rrbracket_{\Gamma+\Theta}, \pi_{i+1}^{m}, \ldots, \pi_{m}^{m}\right\rangle .
$$

If $i=m$, this becomes

$$
\llbracket M\left[x_{m}:=N\right] \rrbracket_{\Gamma+\Theta}=\llbracket M \rrbracket_{\Gamma+\Theta} \circ\left\langle\pi, \llbracket N \rrbracket_{\Gamma+\Theta}\right\rangle,
$$

and if $x_{m} \notin F V(N)$, one has

$$
\llbracket M\left[x_{m}:=N\right] \rrbracket_{\Gamma+\Theta-x_{m}: \tau_{m}}=\llbracket M \rrbracket_{\Gamma+\Theta} \circ\left\langle\mathrm{id}, \llbracket N \rrbracket_{\Gamma+\Theta-x_{m}: \tau_{m}}\right\rangle .
$$

(4) $\llbracket(\lambda x: \sigma . P) Q \rrbracket_{\Gamma+\Theta}=\llbracket P[x:=Q] \rrbracket_{\Gamma+\Theta}$.

Proof. (1) and the first statement of (2) are proved by induction on the derivation of $M$, using lemma 2.1.1 (2)+(3); as for the second statement of (2), if $x_{m} \notin F V(M)$, then

$$
\begin{aligned}
\llbracket M \rrbracket_{\Gamma+\Theta} & =\llbracket M \rrbracket_{\Gamma+\Theta-x_{m}: \tau_{m}} \circ\left\langle\pi_{0}^{m}, \ldots, \pi_{m-1}^{m}\right\rangle \\
& =\llbracket M \rrbracket_{\Gamma+\Theta-x_{m}: \tau_{m}} \circ\left\langle\pi_{0}^{m-1}, \ldots, \pi_{m-1}^{m-1}\right\rangle \circ \pi \\
& =\llbracket M \rrbracket_{\Gamma+\Theta-x_{m}: \tau_{m}} \circ \pi, \quad \text { by (i). }
\end{aligned}
$$


(3) The first statement is proved by induction on the derivation of $M$, using (2), in case $M \equiv \lambda x: \sigma . P$. The rest is easy.

$$
\begin{aligned}
\llbracket(\lambda x: \sigma . P) Q \rrbracket_{\Gamma+\Theta} & =e v \circ\left\langle\Lambda\left(\llbracket P \rrbracket_{\Gamma+\Theta, x: \sigma}\right), \llbracket Q \rrbracket_{\Gamma+\Theta}\right\rangle \\
& =e v \circ\left\langle\Lambda\left(\llbracket P \rrbracket_{\Gamma+\Theta, x: \sigma}\right) \circ \pi, \pi^{\prime}\right\rangle \circ\left\langle\mathrm{id} \llbracket Q \rrbracket_{\Gamma+\Theta}\right\rangle \\
& =\llbracket P \rrbracket_{\Gamma+\Theta, x: \sigma} \circ\left\langle\pi, \pi^{\prime}\right\rangle \circ\left\langle\operatorname{id} \llbracket Q \rrbracket_{\Gamma+\Theta}\right\rangle \\
& =\llbracket P[x:=Q] \rrbracket_{\Gamma+\Theta}, \quad \text { by (iii). }
\end{aligned}
$$

Theorem 4.2.3. ('Soundness'.)

(1) If $\Gamma+\Theta \vdash M=N$ in $\lambda 2$, then $\llbracket M \rrbracket_{\Gamma+\Theta}=\llbracket N \rrbracket_{\Gamma+\Theta}$ holds in every $\lambda 2$-algebra.

(2) If $\Gamma+\Theta \vdash M=N$ in $\lambda \eta 2$, then $\llbracket M \rrbracket_{\Gamma+\Theta}=\llbracket N \rrbracket_{\Gamma+\Theta}$ in all $\lambda \eta 2$-algebras.

Proof. (1) By the previous two lemmas.

(2) Because the fibre categories are ccc's and the adjunctions $\pi_{A, \Omega}^{*} \dashv \prod_{A}$ are normal in a $\lambda \eta 2$-algebra, one can use $\Lambda(e v \circ f \times \mathrm{id})=f$ and $\alpha \circ \beta=\mathrm{id}$, which give the $\eta$-rules (mentioned at the end of subsection 2.3).

4.3.

Valuation maps (or environments) for type of term variables assign certain arrows to these variables. They can be used to give an interpretation of types and terms with specific values for the variables involved. This goes as follows.

Types. Let $\Gamma=\left\{\gamma_{1}: *, \ldots, \gamma_{n}: *\right\} \vdash \sigma: *$ again and let $\xi:$ Typevar $\rightarrow \mathbf{B}(t, \Omega)$ be a valuation for type variables. We put

$$
\begin{aligned}
\xi^{\Gamma} & =\left\langle\operatorname{id}_{t}, \xi\left(\gamma_{1}\right), \ldots, \xi\left(\gamma_{n}\right)\right\rangle: t \rightarrow \llbracket \Gamma \rrbracket \quad \text { in } \mathbf{B} \text { and then } \\
\llbracket \sigma \rrbracket_{\xi} & =\llbracket \sigma \rrbracket_{\Gamma} \circ \xi^{\Gamma}: t \rightarrow \Omega .
\end{aligned}
$$

Terms. Let $\Gamma+\Theta \vdash M: \sigma$ for $\Gamma=\left\{\gamma_{1}: *, \ldots, \gamma_{n}: *\right\}$ and $\Theta=\left\{x_{1}: \tau_{1}, \ldots, x_{m}: \tau_{m}\right\}$ be as before. We write $\llbracket \Theta \rrbracket_{\xi}=\xi^{\Gamma *}\left(\llbracket \Theta \rrbracket_{\Gamma}\right)$, which is an object of $H t$. One can prove that

$$
\llbracket \Theta \rrbracket_{\xi}=t \times \phi_{t}\left(\llbracket \tau_{1} \rrbracket_{\xi}\right) \times \ldots \times \phi_{t}\left(\llbracket \tau_{m} \rrbracket_{\xi}\right) .
$$

Let $\rho$ :Termvar $\rightarrow \bigcup_{X}$. Ht $(t, X)$ be a term valuation map such that $\xi, \rho \models \Gamma+\Theta$, i.e. $\rho\left(x_{j}\right) \in H t\left(t, \phi_{t}\left(\llbracket \tau_{j} \rrbracket_{\xi}\right)\right)$. Analogously to $\xi^{\Gamma}$ we take

$$
\begin{aligned}
\rho^{\Theta} & =\left\langle\mathrm{id}_{t}, \rho\left(x_{1}\right), \ldots, \rho\left(x_{m}\right)\right\rangle: t \rightarrow \llbracket \Theta \rrbracket_{\xi} \quad \text { in } H t \text { and } \\
\llbracket M \rrbracket_{\xi+\rho} & =\xi^{\Gamma *}\left(\llbracket M \rrbracket_{\Gamma+\Theta}\right) \circ \rho^{\Theta}: t \rightarrow \phi_{t}\left(\llbracket \tau \rrbracket_{\xi}\right) .
\end{aligned}
$$

4.4 .

One can now ask whether term and type abstraction are uniform with respect to this assignment of values to term and type variables, i.e. whether

$$
\begin{gathered}
\forall a: t \rightarrow \phi_{t}\left(\llbracket \mu \rrbracket_{\xi}\right) \cdot \llbracket M \rrbracket_{\xi+\rho(y:=a)}=\llbracket N \rrbracket_{\xi+\rho(y:=a)} \Rightarrow \llbracket \lambda y: \mu \cdot M \rrbracket_{\xi+\rho}=\llbracket \lambda y: \mu \cdot N \rrbracket_{\xi+\rho} \text { and } \\
\forall u: t \rightarrow \Omega . \llbracket M \rrbracket_{\xi(\gamma:=u)+\rho}=\llbracket N \rrbracket_{\xi(\gamma:=u)+\rho} \Rightarrow \llbracket \lambda \gamma: * . M \rrbracket_{\xi+\rho}=\llbracket \lambda \gamma: * . N \rrbracket_{\xi+\rho}
\end{gathered}
$$

hold. $\lambda 2$-models are defined in such a way that they satisfy these (semantical) $\xi$-rules. 
Theorem 4.4.1. The above $\xi$-rules hold in $\lambda(\eta) 2$-models.

Proof. As to the first rule, suppose one has $\forall a: t \rightarrow \phi_{t}\left(\llbracket \mu \rrbracket_{\xi}\right) . \llbracket M \rrbracket_{\xi+\rho(y:=a)}=\llbracket N \rrbracket_{\xi+\rho(y:=a)}$; we want to prove $\llbracket \lambda y: \mu . M \rrbracket_{\xi+\rho}=\llbracket \lambda y: \mu . N \rrbracket_{\xi+\rho}$. Therefore we use that the category Ht is a $\lambda 1$-model, see subsection 2.2. First we notice that

$$
\begin{aligned}
\llbracket \lambda y: \mu . M \rrbracket_{\xi+\rho} \cdot a & =e v \circ\left\langle\xi^{\Gamma *}\left(\Lambda\left(\llbracket M \rrbracket_{\Gamma+\Theta, y: \mu}\right)\right) \circ \rho^{\Theta}, a\right\rangle \\
& =e v \circ\left\langle\Lambda\left(\xi^{\Gamma *}\left(\llbracket M \rrbracket_{\Gamma+\Theta, y: \mu}\right) \circ \rho^{\Theta}, a\right\rangle\right. \\
& =\xi^{\Gamma *}\left(\llbracket M \rrbracket_{\Gamma+\Theta, y: \mu}\right) \circ\left\langle\rho^{\Theta}, a\right\rangle \\
& =\llbracket M \rrbracket_{\xi+\rho(y:=a)},
\end{aligned}
$$

and so we may conclude by the Meyer-Scott axiom that

$$
1 \cdot \llbracket \lambda y: \mu \cdot M \rrbracket_{\xi+\rho}=1 \cdot \llbracket \lambda y: \mu \cdot N \rrbracket_{\xi+\rho} .
$$

But

$$
\begin{aligned}
& 1 \cdot \llbracket \lambda y: \mu . M \rrbracket_{\xi+\rho}=\Lambda(e v) \circ \Lambda\left(\xi^{\Gamma *}\left(\llbracket M \rrbracket_{\Gamma+\Theta, y: \mu}\right)\right) \circ \rho^{\Theta} \\
& =L\left(e v \circ\left\langle\Lambda\left(\xi^{\Gamma *}\left(\llbracket M \rrbracket_{\Gamma+\Theta, y: \mu}\right)\right) \circ \rho^{\Theta} \circ \pi, \pi^{\prime}\right\rangle\right) \\
& =\Lambda\left(\xi^{\Gamma *}\left(\llbracket M \rrbracket_{\Gamma+\Theta, y: \mu}\right) \circ\left\langle\rho^{\Theta} \circ \pi, \pi^{\prime}\right\rangle\right) \\
& =\Lambda\left(\xi^{\Gamma *}\left(\llbracket M \rrbracket_{\Gamma+\Theta, y: \mu}\right)\right) \circ \rho^{\Theta} \\
& =\llbracket \lambda y: \mu \cdot M \rrbracket_{\xi+\rho} .
\end{aligned}
$$

Hence $\llbracket \lambda y: \mu . M \rrbracket_{\xi+\rho}=\llbracket \lambda y: \mu . N \rrbracket_{\xi+\rho}$.

As for the other rule, suppose we now have $\forall u: t \rightarrow \Omega$. $\llbracket M \rrbracket_{\xi(\gamma ;=u)+\rho}=\llbracket N \rrbracket_{\xi(\gamma ;=u)+\rho}$; our aim is to deduce $\llbracket \lambda \gamma: * . M \rrbracket_{\xi+\rho}=\llbracket \lambda \gamma: * N \rrbracket_{\xi+\rho}$. First,

$$
\begin{aligned}
\llbracket M \rrbracket_{\xi(\gamma:=u)+\rho} & =\left(\xi(\gamma:=u)^{\Gamma, \gamma: *}\right)^{*}\left(\llbracket M \rrbracket_{\Gamma, \gamma: *+\Theta}\right) \circ \rho^{\Theta} \\
& =\langle\xi \Gamma, u\rangle^{*}\left(\llbracket M \rrbracket_{\Gamma, \gamma: *+\Theta}\right) \circ \rho^{\Theta} \\
& =\left\langle\operatorname{id}_{t}, u\right\rangle^{*}\left\{\left(\xi \mathrm{G} \times \operatorname{id}_{\Omega}\right)^{*}\left(\llbracket M \rrbracket_{\Gamma, \gamma: *+\Theta}\right) \circ \pi_{t, \Omega}^{*}\left(\rho^{\Theta}\right)\right\} .
\end{aligned}
$$

Using that $H$ preserves enough points of $\Omega \cong t \times \Omega$, we obtain

$$
\left(\xi^{\Gamma} \times \mathrm{id}_{\Omega}\right)^{*}\left(\llbracket M \rrbracket_{\Gamma, \gamma: *+\Theta}\right) \circ \pi_{t, \Omega}^{*}\left(p^{\Theta}\right)=\left(\xi^{\Gamma} \times \mathrm{id}_{\Omega}\right)^{*}\left(\llbracket N \rrbracket_{\Gamma, \gamma: *+\Theta}\right) \circ \pi_{t, \Omega}^{*}\left(\rho^{\Theta}\right) .
$$

By applying $\alpha(t)$ on both sides, we are done, since

$$
\begin{aligned}
& \alpha(t)\left\{\left(\xi^{\Gamma} \times \mathrm{id}_{\Omega}\right) *\left(\llbracket M \rrbracket_{\Gamma, \gamma: *+\Theta}\right) \circ \pi_{t, \Omega}^{*}\left(\rho^{\Theta}\right)\right\} \\
& =\alpha(t)\left\{\left(\xi^{\Gamma} \times \operatorname{id}_{\Omega}\right)^{*}\left(\llbracket M \rrbracket_{\Gamma, \gamma: *+\Theta}\right)\right\} \circ \rho^{\Theta} \text {, by lemma 2.2.1(2) } \\
& =\xi^{\Gamma *}\left\{\alpha(\llbracket \Gamma \rrbracket)\left(\llbracket M \rrbracket_{\Gamma, \gamma: *+\Theta}\right)\right\} \circ \rho^{\Theta}, \quad \text { by Beck-Chevalley } \\
& =\xi^{\Gamma *}\left(\llbracket \lambda \gamma: * . M \rrbracket_{\Gamma+\Theta}\right) \circ \rho^{\Theta} \\
& =\llbracket \lambda \gamma: * . M \rrbracket_{\xi+\rho} \text {. }
\end{aligned}
$$


4.5 .

Given a $\lambda 2$-algebra $H$, one can extend the calculus $\lambda 2$ to $\lambda 2(H)$ by adding type and term constants from $H$. This is done in the following way. For $a: t \rightarrow \Omega$ in $\mathbf{B}$ a type constant $c_{a}$ is added by the rule $\vdash c_{a}: *$ and for closed types $\sigma$ and arrows $d: t \rightarrow \phi_{t}(\llbracket \sigma \rrbracket)$ in $H t$, a term constant $c_{d}^{\sigma}$ of type $\sigma$ is added by the rule $\vdash c_{d}^{\sigma}: \sigma$. Obviously, $\lambda 2(H)$ can be interpreted in $H$ by putting for $\Gamma=\left\{\gamma_{1}: *, \ldots, \gamma_{n}: *\right\}$ and $\Theta=\left\{x_{1}: \tau_{1}, \ldots, x_{m}: \tau_{m}\right\}, \llbracket c_{a} \rrbracket_{\Gamma}=a \circ \pi_{0}^{n}$ and $\llbracket c_{d}^{\sigma} \rrbracket_{\Gamma+\Theta}=\pi_{0}^{n *}\left(d \circ \pi_{0}^{m}\right)$.

The above substitution lemmas easily extend to the calculus $\lambda 2(H)$.

Next, some results about the interpretation are established. It is shown that arrows in the category $\lambda 2$-ALG preserve the $\lambda 2$-structure. Moreover, special properties of small $\lambda 2$ algebras and $\lambda 2$-models are noticed, which will be used in section 7 .

Lemma 4.5.1. Suppose $\langle R, S\rangle: H \rightarrow H^{\prime}$ in $\lambda 2$-ALG; for $\sigma, M$ from $\lambda 2(H)$ one has

$$
\begin{aligned}
R\left(\llbracket \sigma \rrbracket_{\Gamma}^{H}\right) & =\llbracket \sigma_{R} \rrbracket_{\Gamma}^{H^{\prime}} \\
S_{[\Gamma]}\left(\llbracket M \rrbracket_{\Gamma+\Theta}^{H}\right) & =\llbracket M_{R, S} \rrbracket_{\Gamma+\Theta}^{H^{\prime}},
\end{aligned}
$$

where $\sigma_{R}$ and $M_{R, S}$ in $\lambda 2\left(H^{\prime}\right)$ are obtained from $\sigma$ and $M$ by replacing the constants $c_{a}$ and $c_{d}^{\sigma}$ in $\sigma$ and $M$ by $c_{R a}$ and $c_{S_{t}}^{\sigma_{R}}$.

Proof. By induction on the derivations, using definition 3.1.2.

Lemma 4.5.2. Suppose $\Gamma \vdash \sigma, \tau: *$.

(1) In a small $\lambda 2$-algebra one has

$$
\forall \xi . \llbracket \sigma \rrbracket_{\xi}=\llbracket \tau \rrbracket_{\xi} \Rightarrow \llbracket \sigma \rrbracket_{\Gamma}=\llbracket \tau \rrbracket_{\Gamma} .
$$

(2) If $\langle R, S\rangle: H \rightarrow H^{\prime}$ with $H$ small, one has for $\sigma, \tau$ from $\lambda 2(H)$,

$$
\forall \xi \cdot \llbracket \sigma \rrbracket_{\xi}^{H}=\llbracket \tau \rrbracket_{\xi}^{H} \Rightarrow \forall \xi \cdot \llbracket \sigma_{R} \rrbracket_{\xi}^{H^{\prime}}=\llbracket \tau_{R} \rrbracket_{\xi}^{H^{\prime}} .
$$

Proof. (1) Since $\llbracket \Gamma \rrbracket=t \times \Omega \times \ldots \times \Omega \cong \Omega \times \ldots \times \Omega$ has enough points.

(2) By (1) and lemma 4.5.1(1).

Lemma 4.5.3. Suppose $\Gamma+\Theta \vdash M=N$ : $\sigma$. In a $\lambda 2$-model one has

$$
\forall \xi, \rho \cdot \llbracket M \rrbracket_{\xi+\rho}=\llbracket N \rrbracket_{\xi+\rho} \Rightarrow \llbracket M \rrbracket_{\Gamma+\Theta}=\llbracket N \rrbracket_{\Gamma+\Theta} .
$$

Proof. Assume $\Gamma=\left\{\gamma_{1}: *, \ldots, \gamma_{n}: *\right\}$ and $\Theta=\left\{x_{1}: \tau_{1}, \ldots, x_{m}: \tau_{m}\right\}$. If one has that $\llbracket M \rrbracket_{\xi+\rho}=\llbracket N \rrbracket_{\xi+\rho}$ for all $\xi, \rho$ then by using theorem 4.4.1 $n+m$ times, one obtains $\llbracket \lambda \gamma: * \lambda \boldsymbol{x}: \tau$. $M \rrbracket_{\varnothing+\varnothing}=\llbracket \lambda \gamma: * \lambda \boldsymbol{x}: \tau . N \rrbracket_{\varnothing+\varnothing}$. By soundness we have $\llbracket M \rrbracket_{\Gamma+\Theta}=\llbracket(\lambda \gamma: * \lambda \boldsymbol{x}: \tau . M) \boldsymbol{\gamma} \boldsymbol{x} \rrbracket_{\Gamma+\Theta}$ so we are done.

\section{Second order sums}

In this section we first look at the categorical description of sums and in the last subsection 5.5 at what can be defined syntactically. 
5.1 .

The rules for second order sums are as follows.

$$
\begin{gathered}
\Gamma, \gamma: * \vdash \sigma: * \Rightarrow \Gamma \vdash \exists \gamma: *, \sigma: * \\
\Gamma \vdash \tau: *, \Gamma+\Theta \vdash M: \sigma[\gamma:=\tau] \Rightarrow \Gamma+\Theta \vdash\langle\tau, M\rangle: \exists \gamma: *, \sigma \\
\Gamma+\Theta \vdash P: \exists \gamma: * . \sigma, \Gamma \vdash \mu: *, \\
\Gamma, \gamma: *+\Theta, x: \sigma \vdash Q: \mu \quad \text { with } \quad \gamma \notin F V(\Theta) \Rightarrow \Gamma+\Theta \vdash \mathscr{E}_{\gamma: *, x: \sigma}(P Q): \mu,
\end{gathered}
$$

where $\mathscr{E}_{\gamma: *, x: \sigma}$ is a constant that binds the variables $\gamma, x$ in $Q$ (usually, we abbreviate the subscript as $\gamma, x$ or sometimes we omit it altogether). The ensuing conversion rules are

$$
\begin{aligned}
\mathscr{E}_{\gamma, x}(\langle\tau, M\rangle, Q) & =Q[\gamma:=\tau][x:=M] \quad(\beta) \\
\mathscr{E}_{\gamma, x}(P,\langle\gamma, x\rangle) & =P \quad(\eta)
\end{aligned}
$$

Furthermore, the following commutation or permutation conversion is required. If $\Gamma+\Theta, y: \mu \vdash N: \rho$, then

$$
N\left[y:=\mathscr{E}_{\gamma, x}(P, Q)\right]=\mathscr{E}_{\gamma, x}(P, N[y:=Q]),
$$

see also Prawitz $(1965$, IV, $\S 1)$, Seely $(1983, \S 2)$ or Girard et al. $(1989,10.4)$. It will be of crucial importance in 5.5 and 6.1 .

Definition 5.2. Let $H$ be a $\lambda 2$-algebra as described in definition $3.1 ; H$ admits sums if there are semi-adjunctions $\Sigma_{A} \dashv_{s} \pi_{A, \Omega}^{*}$ which satisfy a Beck-Chevalley condition: for $u: B \rightarrow A$ in $\mathbf{B}$ one has that $\left\langle\left(u \times \mathrm{id}_{\Omega}\right)^{*}, u^{*}\right\rangle$ is a map from the semi-adjunction $\Sigma_{A} \dashv_{s} \pi_{A, \Omega}^{*}$ to $\Sigma_{B} \dashv_{s} \pi_{B, \Omega}^{*}$.

\section{3.}

The interpretation of the sums as described above appears to be a non-trivial exercise in a non-extensional setting. First, we have to investigate what can be used of the Frobenius reciprocity. Therefore we consider a semi-adjunction $F \dashv_{s} G$ (as described in subsection 2.1) between two semi-ccc's $\mathbf{C}, \mathbf{D}$ such that $G$ is a normal functor that preserves the semi-ccc structure. Dually to lemma 2.1.1, one can now prove that $\alpha \circ \beta=$ id and also that $\alpha(u \circ v)=G u \circ \alpha(v), \alpha(u \circ F v)=\alpha(u) \circ v, \beta(G u \circ v)=u \circ \beta(v)$ and $\beta(u) \circ F v=\beta(u \circ v)$.

Lemma 5.3.1. ('Non-extensional Frobenius'.)

(1) There is a natural transformation

$$
\kappa_{Z}: \mathbf{D}(F(G Y \times X), Z) \rightarrow \mathbf{D}(Y \times F X, Z) .
$$

The dependence of $\kappa_{Z}$ on $X, Y$ is left implicit. Especially, one has

$$
\psi_{X, Y}=\kappa_{F(G Y \times X)}(\mathrm{id}): Y \times F X \rightarrow F(G Y \times X) .
$$

(2) $\psi_{X, Y}$ is natural in $Y$, i.e. for $f: Y \rightarrow Y^{\prime}$ one has

$$
\psi_{X, Y} \circ f \times \mathrm{id}=F(G f \times \mathrm{id}) \circ \psi_{X, Y} .
$$


(3) If we write $\chi=\beta(G \mathrm{id} \times \alpha(\mathrm{id})): F(G Y \times X) \rightarrow Y \times F X$, then one has

$$
\alpha(\psi \circ \chi)=\alpha(\mathrm{id}) \circ\left\langle\pi, \pi^{\prime}\right\rangle
$$

and

$$
\chi \circ \psi=\left\langle\pi, \pi^{\prime}\right\rangle \quad \text { in case } \beta \circ \alpha=\mathrm{id},
$$

i.e. the latter equality holds in case one has a normal adjunction $F \dashv G$. The first equality expresses that $\chi$ is some sort of right inverse to $\psi$, see the proof of proposition $5.4 .2(1)$ below.

Proof. (1)

$$
\begin{array}{ccc} 
& f: F(G Y \times X) \rightarrow Z, \\
\Rightarrow & \alpha(f): G Y \times X \rightarrow G Z \\
\Rightarrow & \Lambda\left(\alpha(f) \circ\left\langle\pi^{\prime}, \pi\right\rangle\right): X \rightarrow G Z^{G Y}=G\left(Z^{Y}\right) \\
\Rightarrow & & \beta\left(\Lambda\left(\alpha(f) \circ\left\langle\pi^{\prime}, \pi\right\rangle\right)\right): F X \rightarrow Z^{Y} \\
\Rightarrow & \kappa_{Z}(f)=e v \circ\left\langle\beta\left(\Lambda\left(\alpha(f) \circ\left\langle\pi^{\prime}, \pi\right\rangle\right)\right) \circ \pi^{\prime}, \pi\right\rangle: Y \times F X \rightarrow Z .
\end{array}
$$

Since all these transitions are natural in $Z$, so is their composition $\kappa_{Z}$. It means that for $h: Z \rightarrow Z^{\prime}$ one has $h \circ \kappa_{Z}(f)=\kappa_{Z}(h \circ f)$.

(2) $F(G f \times$ id $) \circ \psi_{X, Y}=F(G f \times$ id $) \circ \kappa($ id $)=\kappa(F(G f \times$ id $))$, by naturality

$$
\begin{aligned}
& =e v \circ\left\langle\beta \Lambda\left\{\alpha(F(G f \times \mathrm{id})) \circ\left\langle\pi^{\prime}, \pi\right\rangle\right\} \circ \pi^{\prime}, \pi\right\rangle \\
& =e v \circ\left\langle\beta \Lambda\left\{\alpha(\mathrm{id}) \circ G f \times \mathrm{id} \circ\left\langle\pi^{\prime}, \pi\right\rangle\right\} \circ \pi^{\prime}, \pi\right\rangle \\
& =e v \circ\left\langle\beta \Lambda\left\{\alpha(\mathrm{id}) \circ\left\langle\pi^{\prime}, \pi\right\rangle \circ\left\langle\pi, G f \circ \pi^{\prime}\right\rangle\right\} \circ \pi^{\prime}, \pi\right\rangle \\
& =e v \circ\left\langle\beta \Lambda\left\{e v \circ\left\langle\Lambda\left(\alpha(\mathrm{id}) \circ\left\langle\pi^{\prime}, \pi\right\rangle\right) \circ \pi, G f \circ \pi^{\prime}\right\rangle\right\} \circ \pi^{\prime}, \pi\right\rangle \\
& =e v \circ\left\langle\beta \Lambda\left\{e v \circ\left\langle\pi, G f \circ \pi^{\prime}\right\rangle \circ\left\langle\Lambda\left(\alpha(\mathrm{id}) \circ\left\langle\pi^{\prime}, \pi\right\rangle\right) \circ \pi, \pi^{\prime}\right\rangle\right\} \circ \pi^{\prime}, \pi\right\rangle \\
& =e v \circ\left\langle\beta\left\{\Lambda\left(e v \circ\left\langle\pi, G f \circ \pi^{\prime}\right\rangle\right) \circ \Lambda\left(\alpha(\mathrm{id}) \circ\left\langle\pi^{\prime}, \pi\right\rangle\right)\right\} \circ \pi^{\prime}, \pi\right\rangle \\
& =e v \circ\left\langle\beta\left\{G \Lambda\left(e v \circ\left\langle\pi, f \circ \pi^{\prime}\right\rangle\right) \circ \Lambda\left(\alpha(\mathrm{id}) \circ\left\langle\pi^{\prime}, \pi\right\rangle\right)\right\} \circ \pi^{\prime}, \pi\right\rangle \\
& =e v \circ\left\langle\Lambda\left(e v \circ\left\langle\pi, f \circ \pi^{\prime}\right\rangle\right) \circ \beta \Lambda\left(\alpha(\mathrm{id}) \circ\left\langle\pi^{\prime}, \pi\right\rangle\right) \circ \pi^{\prime}, \pi\right\rangle \\
& =e v \circ\left\langle\pi, f \circ \pi^{\prime}\right\rangle \circ\left\langle\beta \Lambda\left(\alpha(\mathrm{id}) \circ\left\langle\pi^{\prime}, \pi\right\rangle\right) \circ \pi^{\prime}, \pi\right\rangle \\
& =e v \circ\left\langle\beta \Lambda\left(\alpha(\mathrm{id}) \circ\left\langle\pi^{\prime}, \pi\right\rangle\right) \circ \pi^{\prime}, f \circ \pi\right\rangle \\
& =\psi_{X, Y^{\prime}} \circ f \times \mathrm{id} .
\end{aligned}
$$


(3) $\alpha(\psi \circ \chi)=G \psi \circ \alpha(\chi)=G \psi \circ G$ id $\times \alpha($ id $)$

$$
\begin{aligned}
& =e v \circ\left\langle G \beta \Lambda\left(\alpha(\mathrm{id}) \circ\left\langle\pi^{\prime}, \pi\right\rangle\right) \circ \alpha(\mathrm{id}) \circ \pi^{\prime}, \pi\right\rangle \\
& =e v \circ\left\langle\alpha \beta \Lambda\left(\alpha(\mathrm{id}) \circ\left\langle\pi^{\prime}, \pi\right\rangle\right) \circ \pi^{\prime}, \pi\right\rangle \\
& =e v \circ\left\langle\Lambda\left(\alpha(\mathrm{id}) \circ\left\langle\pi^{\prime}, \pi\right\rangle\right) \circ \pi^{\prime}, \pi\right\rangle \\
& =\alpha(\mathrm{id}) \circ\left\langle\pi^{\prime}, \pi\right\rangle \circ\left\langle\pi^{\prime}, \pi\right\rangle \\
& =\alpha(\mathrm{id}) \circ\left\langle\pi, \pi^{\prime}\right\rangle .
\end{aligned}
$$

Analogously, one can prove that $\chi \circ \psi=\left\langle\pi, \pi^{\prime}\right\rangle$ if $\beta \circ \alpha=$ id.

One more technical result is needed, before we can describe the interpretation; the setting in the next lemma is still as above.

Lemma 5.3.2. For $f: G Y \times X \rightarrow G Z$ one has

$$
\langle\pi, \beta(f) \circ \psi\rangle=\left\langle\pi, \pi^{\prime}\right\rangle \circ \beta(\langle\pi, f\rangle) \circ \psi
$$

Proof. One easily verifies that $\beta(f) \circ \psi=\pi^{\prime} \circ \beta(\langle\pi, f\rangle) \circ \psi$. For $\pi=\pi \circ \beta(\langle\pi, f\rangle) \circ \psi$ one uses the naturality of $\kappa$ in $\psi=\kappa(\mathrm{id})$ and $\beta\left(\Lambda\left(\pi_{X, G Y}^{\prime}\right)\right)=\Lambda\left(\pi_{F X, Y}^{\prime}\right)$. The latter holds since

$$
\begin{aligned}
\beta\left(\Lambda\left(\pi_{X, G Y}^{\prime}\right)\right) & =\beta\left\{\Lambda\left(\pi_{G F X, G Y}^{\prime}\right) \circ \alpha\left(\mathrm{id}_{F X}\right)\right\} \\
& =\beta\left\{G \Lambda\left(\pi_{F X, Y}^{\prime}\right) \circ \alpha\left(\mathrm{id}_{F X}\right)\right\} \\
& =\Lambda\left(\pi_{F X, Y}^{\prime}\right) \circ \beta\left(\alpha\left(\mathrm{id}_{F X}\right)\right) \\
& =\Lambda\left(\pi_{F X, Y}^{\prime}\right) .
\end{aligned}
$$

5.4 .

Next we shall describe the interpretation of the sum rules as described in the beginning of this section. We start with a $\lambda 2$-algebra (as defined in definition 3.1) that admits sums.

- If $\Gamma, \gamma: * \vdash \sigma: *$, then

$$
\llbracket \exists \gamma: * . \sigma \rrbracket_{\Gamma}=\phi_{[\Gamma \rrbracket}^{-1}\left(\Sigma_{\llbracket \Gamma]}\left(\phi_{[\Gamma, \gamma: *]}\left(\llbracket \sigma \rrbracket_{\Gamma, \gamma: *}\right)\right)\right),
$$

analogously to the definition of $\llbracket \forall \gamma: * . \sigma \rrbracket_{\Gamma}$ in 4.1 .

- If $\Gamma \vdash \tau: *$ and $\Gamma+\Theta \vdash M: \sigma[\gamma:=\tau]$, then

$$
\left.\llbracket\langle\tau, M\rangle \rrbracket_{\Gamma+\Theta}=\left\langle\mathrm{id}, \llbracket \tau \rrbracket_{\Gamma}\right\rangle *(\alpha \llbracket \Gamma \rrbracket)(\mathrm{id})\right) \circ \llbracket M \rrbracket_{\Gamma+\Theta} .
$$

- If $\Gamma+\Theta \vdash P: \exists \gamma: * . \sigma, \Gamma \vdash \mu: *, \Gamma, \gamma: *+\Theta, x: \sigma \vdash Q: \mu$ with $\gamma \notin F V(\Theta)$, then

$$
\llbracket \mathscr{E}_{\gamma, x}(P, Q) \rrbracket_{\Gamma+\Theta}=\beta(\llbracket \Gamma \rrbracket)\left(\llbracket Q \rrbracket_{\Gamma, \gamma: *+\Theta, x: \sigma}\right) \circ \psi \circ\left\langle\mathrm{id}, \llbracket P \rrbracket_{\Gamma+\Theta}\right\rangle,
$$

where $\psi$ is as determined in lemma 5.3.1(1).

Lemma 5.4.1. (1) The substitution lemmas 4.1.1, 4.2.1 and 4.2.2 also apply to the above interpretation of sums.

(2) The commutation conversion holds, i.e.

$$
\llbracket N\left[y:=\mathscr{E}_{\gamma, x}(P, Q)\right] \rrbracket_{\Gamma+\Theta}=\llbracket \mathscr{E}_{\gamma, x}(P, N[y:=Q]) \rrbracket_{\Gamma+\Theta} .
$$


Proof. (1) Most of this is straightforward, except for the first statements from points 4.2.2(2) and (3) applied to the term $\mathscr{E}_{\gamma, x}(P, Q)$. Then one has to use lemma 5.3.1(2).

(2) By lemma 5.3.2.

As can be expected by now, the semi-adjunction $\Sigma_{A} \dashv_{s} \pi_{A, \Omega}^{*}$ makes the $\beta$-conversion rule valid, but not the $\eta$-rule; therefore a normal adjunction is needed.

Proposition 5.4.2. The above interpretation yields

(1) $\llbracket \mathscr{E}_{\gamma, x}(\langle\tau, M\rangle, Q) \rrbracket_{\Gamma+\Theta}=\llbracket Q[\gamma:=\tau][x:=M] \rrbracket_{\Gamma+\Theta}$.

(2) $\llbracket \mathscr{E}_{\gamma, x}(P,\langle\gamma, x\rangle) \rrbracket_{\Gamma+\Theta}=\llbracket P \rrbracket_{\Gamma+\Theta} \quad$ in case one has normal adjunctions $\Sigma_{A} \dashv \pi_{A, \Omega}^{*}$.

Proof. (1)

$\llbracket \mathscr{E}_{\gamma, x}(\langle\tau, M\rangle, Q) \rrbracket_{\Gamma+\Theta}$

$\left.=\beta(\llbracket \Gamma \rrbracket)\left(\llbracket Q \rrbracket_{\Gamma, \gamma: *+\Theta, x: \sigma}\right) \circ \psi \circ\left\langle\mathrm{id},\left\langle\mathrm{id}, \llbracket \tau \rrbracket_{\Gamma}\right\rangle^{*}(\alpha \llbracket \Gamma \rrbracket)(\mathrm{id})\right) \circ \llbracket M \rrbracket_{\Gamma+\Theta}\right\rangle$

$=\beta\left(\_\right) \circ \psi \circ\left\langle\mathrm{id}, \llbracket \tau \rrbracket_{\Gamma}\right\rangle^{*}\{\mathrm{id} \times \alpha(\llbracket \Gamma \rrbracket)(\mathrm{id})\} \circ\left\langle\mathrm{id}, \llbracket M \rrbracket_{\Gamma+\Theta}\right\rangle$

$=\left\langle\mathrm{id}, \llbracket \tau \rrbracket_{\Gamma}\right\rangle^{*}\left\{\pi^{*}\left(\beta\left(\_\right) \circ \psi\right) \circ \mathrm{id} \times \alpha(\llbracket \Gamma \rrbracket)(\mathrm{id})\right\} \circ\langle-\rangle$

$=\left\langle\mathrm{id}, \llbracket \tau \rrbracket_{\Gamma}\right\rangle^{*}\left\{\pi^{*}\left(\beta\left(\_\right)\right) \circ \pi^{*}(\psi) \circ \alpha(\llbracket \Gamma \rrbracket)(\chi)\right\} \circ\langle-\rangle$,

see the definition of $\chi$ in lemma 5.3.1(3)

$=\left\langle\mathrm{id}, \llbracket \tau \rrbracket_{\Gamma}\right\rangle^{*}\left\{\pi^{*}\left(\beta\left(\_\right)\right) \circ \alpha(\llbracket \Gamma \rrbracket)(\mathrm{id}) \circ\left\langle\pi, \pi^{\prime}\right\rangle\right\} \circ\langle-\rangle$,

since $\pi^{*}(\psi) \circ \alpha(\llbracket \Gamma \rrbracket)(\chi)=\alpha(\llbracket \Gamma \rrbracket)(\psi \circ \chi)=\alpha(\llbracket \Gamma \rrbracket)(\mathrm{id}) \circ\left\langle\pi, \pi^{\prime}\right\rangle$, by lemma 5.3.1(3) again

$\left.=\left\langle\mathrm{id}, \llbracket \tau \rrbracket_{\Gamma}\right\rangle^{*}\left\{\alpha(\llbracket \Gamma \rrbracket) \circ \beta(\llbracket \Gamma \rrbracket)\left(\llbracket Q \rrbracket_{\Gamma, \gamma: *+\Theta, x: \sigma}\right)\right) \circ\left\langle\pi, \pi^{\prime}\right\rangle\right\} \circ\langle-\rangle$

$=\left\langle\mathrm{id}, \llbracket \tau \rrbracket_{\Gamma}\right\rangle^{*}\left\{\llbracket Q \rrbracket_{\Gamma, \gamma: *+\Theta, x: \sigma}\right\} \circ\left\langle\mathrm{id}, \llbracket M \rrbracket_{\Gamma+\Theta}\right\rangle$

$=\llbracket Q[\gamma:=\tau][x:=M] \rrbracket_{\Gamma+\Theta}$,

by lemma 5.4.1(1).

(2)

$$
\begin{aligned}
\llbracket \mathscr{E}_{\gamma, x} & (P,\langle\gamma, x\rangle) \rrbracket_{\Gamma+\Theta} \\
& =\beta(\llbracket \Gamma \rrbracket)\left(\llbracket\langle\gamma, x\rangle \rrbracket_{\Gamma, \gamma: *+\Theta, x: \sigma}\right) \circ \psi \circ\left\langle\mathrm{id}, \llbracket P \rrbracket_{\Gamma+\Theta}\right\rangle \\
& =\beta(\llbracket \Gamma \rrbracket)\left\{\left\langle\mathrm{id}, \llbracket \gamma \rrbracket_{\Gamma, \gamma: *}\right\rangle^{*} \alpha(\llbracket \Gamma, \gamma: * \rrbracket)(\mathrm{id}) \circ \llbracket x \rrbracket_{\Gamma, \gamma: *+\Theta, x: \sigma}\right\} \circ \psi \circ\left\langle_{-}\right\rangle \\
& =\beta(\llbracket \Gamma \rrbracket)\left\{\left\langle\mathrm{id}, \pi^{\prime}\right\rangle^{*} \alpha(\llbracket \Gamma, \gamma: * \rrbracket)\left(\pi^{*}(\mathrm{id})\right) \circ \pi^{\prime}\right\} \circ \psi \circ\langle-\rangle \\
& =\beta(\llbracket \Gamma \rrbracket)\left\{\left\langle\mathrm{id}, \pi^{\prime}\right\rangle^{*}(\pi \times \mathrm{id})^{*} \alpha(\llbracket \Gamma \rrbracket)(\mathrm{id}) \circ \pi^{\prime}\right\} \circ \psi \circ\left\langle\left\langle_{-}\right\rangle\right. \\
& =\beta(\llbracket \Gamma \rrbracket)\left\{\alpha(\llbracket \Gamma \rrbracket)(\mathrm{id}) \circ \pi^{\prime}\right\} \circ \psi \circ\langle-\rangle \\
& =\beta(\llbracket \Gamma \rrbracket)\left\{\pi^{*}\left(\pi^{\prime}\right) \circ \pi^{*}(\mathrm{id}) \times \alpha(\llbracket \Gamma \rrbracket)(\mathrm{id})\right\} \circ \psi \circ\langle-\rangle \\
& =\pi^{\prime} \circ \beta(\llbracket \Gamma \rrbracket)\left\{\pi^{*}(\mathrm{id}) \times \alpha(\llbracket \Gamma \rrbracket)(\mathrm{id})\right\} \circ \psi \circ\langle-\rangle \\
& =\pi^{\prime} \circ \chi \circ \psi \circ\langle-\rangle, \quad \text { see lemma } 5.3 .1(3) \\
& =\pi^{\prime} \circ\left\langle\pi, \pi^{\prime}\right\rangle \circ\left\langle\mathrm{id}, \llbracket P \rrbracket_{\Gamma+\Theta}\right\rangle \\
& =\llbracket P \rrbracket_{\Gamma+\Theta} .
\end{aligned}
$$


5.5 .

In the calculus $\lambda 2$ one can define 'pseudo-sums' as follows.

$$
\begin{aligned}
\exists \gamma: * . \sigma & \equiv \forall \beta: * .(\forall \gamma: * .(\sigma \rightarrow \beta)) \rightarrow \beta \\
\langle\tau, M\rangle & \equiv \lambda \beta: * . \lambda z: \forall \gamma: * .(\sigma \rightarrow \beta) . z \tau M \\
\mathscr{E}_{\gamma, x}(P, Q) & \equiv P \mu(\lambda \gamma: * . \lambda x: \sigma \cdot Q) .
\end{aligned}
$$

Indeed,

$$
\begin{aligned}
\mathscr{E}_{\gamma, x}(\langle\tau, M\rangle, Q) & =\langle\tau, M\rangle \mu(\lambda \gamma: * . \lambda x: \sigma . Q) \\
& =(\lambda \gamma: * . \lambda x: \sigma . Q) \tau M \\
& =Q[\gamma:=\tau][x:=M],
\end{aligned}
$$

but the commutation conversion

$$
N\left[y:=\mathscr{E}_{\gamma, x}(P, Q)\right]=\mathscr{E}_{\gamma, x}(P, N[y:=Q])
$$

does not hold. For this reason we speak of pseudo-sums. In 6.1 in the next section, we shall show that sums as described in 5.1 (i.e. with the commutation conversion) give rise to left semi-adjoints to the $\pi^{*}$ functors in a term model, which proves the 'completeness' (of the sums from 5.1). Hence we conclude that the above syntactical definitions do not provide $\lambda 2$ with proper sums.

\section{Examples}

In this section we describe six different hyperdoctrine models: two term models (a $\lambda 2-$ algebra and a $\lambda 2$-model), three ideal models (two $\lambda 2$-algebras and one $\lambda 2$-model) and the PER-model (a $\lambda \eta 2$-model). Our aim is to give (simple) examples of the previously defined notions - not to plunge into semantic technicalities.

Quite recently, in Hoofman (1990), a non-extensional $\lambda 2$-model was obtained by using coherence spaces with continuous functions instead of stable ones.

\section{1 .}

The following construction of a term model of $\lambda 2$ is standard, see e.g. Seely $(1987,4.5)$ or Pitts $(1987,2.6)$; it will be used again in section 7 . We begin by fixing the collection of type variables as $\left\{\gamma_{n} \mid n \in \omega\right\}$ and by choosing for every type $\sigma$ a (fixed) term variable $x^{\sigma}$ of type $\sigma$. Let's write $\Gamma_{m}=\left\{\gamma_{1}: *, \ldots, \gamma_{m}: *\right\}$.

A base category $\mathbf{B}$ is defined with objects $n \in \omega$ and with morphisms $\left(\sigma_{1}, \ldots, \sigma_{n}\right): m \rightarrow n$ determined by types $\sigma_{i}$ with $\Gamma_{m} \vdash \sigma_{i}: *$. Composition is done by (simultaneous) substitution and $\mathrm{id}_{n}=\left(\gamma_{1}, \ldots, \gamma_{n}\right)$. Furthermore, $n+m$ is the product of $n, m \in \mathbf{B}$ (with $\pi=\left(\gamma_{1}, \ldots, \gamma_{n}\right)$ and $\left.\pi^{\prime}=\left(\gamma_{n+1}, \ldots, \gamma_{n+m}\right)\right)$ and hence 0 is terminal. As distinguished object we take $\Omega=1 \in \mathbf{B}$.

A functor $H: \mathbf{B}^{\text {op }} \rightarrow$ Cat is defined on objects $n \in \mathbf{B}$ as follows. The category $H n$ has arrows $\sigma: n \rightarrow 1$ from $\mathbf{B}$ as objects; morphisms $[M]: \sigma \rightarrow \tau$ are $\beta$-equivalence classes of terms $M$ with $\Gamma_{n}+x^{\sigma}: \sigma \vdash M: \tau$, where $[M]=\left\{N \mid \Gamma_{n}+x^{\sigma}: \sigma \vdash M=N: \tau\right\}$. Again composition is done by 
substitution and $\mathrm{id}_{\sigma}=\left[x^{\sigma}\right]$. For $\left(\mu_{1}, \ldots, \mu_{n}\right): m \rightarrow n$ in $\mathbf{B}$, we define the functor $\left.\left(\mu_{1}, \ldots, \mu_{n}\right)\right)^{*}$ : $H n \rightarrow H m$ by

$$
\begin{aligned}
\left(\mu_{1}, \ldots, \mu_{n}\right)^{*}(\sigma) & =\sigma \circ\left(\mu_{1}, \ldots, \mu_{n}\right)=\sigma\left[\gamma_{1}:=\mu_{1}, \ldots, \gamma_{n}:=\mu_{n}\right] \\
\left(\mu_{1}, \ldots, \mu_{n}\right)^{*}([M]) & =\left[M\left[\gamma_{1}:=\mu_{1}, \ldots, \gamma_{n}:=\mu_{n}\right]\right] .
\end{aligned}
$$

The categories $H n$ have a semi-ccc structure. For the semi-terminal object one can take any closed inhabited type, say $s t=\forall \alpha: * .(\alpha \rightarrow \alpha)$. Then $!_{\sigma}=[\lambda \alpha: * \lambda x: \alpha . x]: \sigma \rightarrow s t$. The semiproduct is given by the canonical pairing definable in $\lambda 2$, i.e. $\sigma \times \tau \equiv \forall \beta: * .((\sigma \rightarrow \tau \rightarrow \beta) \rightarrow \beta)$, where $\beta$ is fresh. For terms $M: \sigma$ and $N: \tau$, we then have $\langle\langle M, N\rangle\rangle \equiv \lambda \beta: * \lambda z: \sigma \rightarrow \tau \rightarrow \beta$. $z M N: \sigma \times \tau$ and $p \equiv \lambda w: \sigma \times \tau . \quad w \sigma(\lambda x: \sigma \lambda y: t . x)$ and $p^{\prime} \equiv \lambda w: \sigma \times \tau . \quad w \tau(\lambda x: \sigma \lambda y: \tau . y)$; thus $p\langle\langle M, N\rangle\rangle=M$ and $p^{\prime}\langle\langle M, N\rangle\rangle=N$. Hence we take in the category $H n,\langle[M],[N]\rangle$ $=[\langle\langle M, N\rangle\rangle]$ and $\pi=\left[p x^{\sigma \times r}\right], \pi^{\prime}=\left[p^{\prime} x^{\sigma \times \tau}\right]$.

The semi-exponent is given by $\tau^{\sigma}=\sigma \rightarrow \tau$ with for $[M]: \mu \times \sigma \rightarrow \tau$, the exponential adjunct $\Lambda([M])=\left[\lambda z: \sigma \cdot M\left[x^{\mu \times \sigma}:=\left\langle\left\langle x^{\mu}, z\right\rangle\right\rangle\right]\right]: \mu \rightarrow \tau^{\sigma}$ and $e v=\left[\left(p x^{\tau^{\sigma} \times \sigma}\right)\left(p^{\prime} x^{\tau^{\sigma} \times \sigma}\right)\right]$. By using the calculus $\lambda \eta 2$ one obtains $\Lambda(e v)=\mathrm{id}$, but not $\left\langle\pi, \pi^{\prime}\right\rangle=\mathrm{id}$ or $!_{s t}=\mathrm{id}_{s t}$. So in that case one would not have a ccc.

Product semi-functors $\Pi_{n}: H(n+1) \rightarrow H n$ can be defined by

$$
\begin{aligned}
\sigma & \mapsto \forall \gamma_{n+1}: * . \sigma \\
{[M]: \sigma \rightarrow \tau } & \mapsto\left[\lambda \gamma_{n+1}: * . M\left[x^{\sigma}:=x^{\Pi_{n}(\sigma)} \gamma_{n+1}\right]\right] .
\end{aligned}
$$

The maps $H(n+1)\left(\pi_{n, 1}(\tau), \sigma\right) \rightleftarrows H(n)\left(\tau, \Pi_{n}(\sigma)\right)$ are given by

$$
\begin{aligned}
{[M] } & \mapsto\left[\lambda \gamma_{n+1}: * . M\right] \\
{\left[N \gamma_{n+1}\right] } & \leftrightarrow[N] .
\end{aligned}
$$

In the calculus $\lambda \eta 2$ one obtains a functor $\Pi_{n}$ and an adjunction $\pi_{n, 1}^{*} \dashv \Pi_{n}$.

In case the language contains sums as described in 5.1, semi-functors $\Sigma_{n}: H(n+1) \rightarrow H n$ can be defined analogously.

$$
\begin{aligned}
\sigma & \mapsto \exists \gamma_{n+1}: * . \sigma \\
{[M]: \sigma \rightarrow \tau } & \mapsto\left[\mathscr{E}_{\gamma_{n+1}, x^{\sigma}}\left(x^{\Sigma_{n}(\sigma)},\left\langle\gamma_{n+1}, M\right\rangle\right)\right] .
\end{aligned}
$$

We check that $\Sigma_{n}$ is a semi-functor (omitting irrelevant details).

$$
\begin{aligned}
\Sigma_{n}([N]) \circ \Sigma_{n}([M]) & =\left[\mathscr{E}\left(x^{\prime},\langle\gamma, N\rangle\right)\left[x^{\prime}:=\mathscr{E}(x,\langle\gamma, M\rangle)\right]\right] \\
& =\left[\mathscr{E}\left(x, \mathscr{E}\left(x^{\prime},\langle\gamma, N\rangle\right)\left[x^{\prime}:=\langle\gamma, M\rangle\right]\right)\right], \quad \text { by commutation } \\
& =[\mathscr{E}(x, \mathscr{E}(\langle\gamma, M\rangle,\langle\gamma, N\rangle))] \\
& =\left[\mathscr{E}\left(x,\left\langle\gamma, N\left[x^{\tau}:=M\right]\right\rangle\right)\right], \quad \text { by }(\beta) \\
& =\Sigma_{n}([N] \circ[M]) .
\end{aligned}
$$

The maps $H(n)\left(\Sigma_{n}(\sigma), \tau\right) \rightleftarrows H(n+1)\left(\sigma, \pi_{n, 1}^{*}(\tau)\right)$ are given by

$$
\begin{aligned}
{[M] } & \mapsto\left[M\left[x^{\Sigma_{n}(\sigma)}:=\left\langle\gamma_{n+1}, x^{\sigma}\right\rangle\right]\right] \\
{\left[\mathscr{E}_{\gamma_{n+1}, x^{\sigma}}\left(x^{\Sigma_{n}(\sigma)}, N\right)\right] } & \leftrightarrow[N] .
\end{aligned}
$$


In case one has $\eta$ for sums, $\Sigma_{n}$ is a functor and these maps are each other's inverses; thus an adjunction is obtained.

\section{2.}

A second term model can be obtained by defining a different functor $H: \mathbf{B}^{\text {op }} \rightarrow$ Cat on the same basis. In $H n$ we now take

$$
\begin{array}{ll}
\text { obj. } & \Lambda_{\sigma}=\left\{[M] \mid \exists \Theta . \Gamma_{n}+\Theta \vdash M: \sigma\right\} \text { for } \sigma: n \rightarrow 1 \text { in } \mathbf{B} . \\
\text { mor. } & f: \Lambda_{\sigma} \rightarrow \Lambda_{\tau}
\end{array}
$$

are maps which are realised by a certain term $Q$ of type $\sigma \rightarrow \tau$, i.e.

$$
\forall[M] \in \Lambda_{\sigma} . f([M])=[Q M] .
$$

The identity map on $\Lambda_{\sigma}$ is then realised by $I_{\sigma}=\lambda x: \sigma \cdot x$ and the composition $g \circ f$ by $\lambda z: \sigma \cdot Q_{g}\left(Q_{f} z\right)$, where $Q_{g}, Q_{f}$ are realisers of $g, f$. Furthermore,

$$
\begin{aligned}
\left(\mu_{1}, \ldots, \mu_{n}\right)^{*}\left(\Lambda_{\sigma}\right) & =\Lambda_{\sigma\left[\gamma_{1}:=\mu_{1}, \ldots, \gamma_{n}:=\mu_{n}\right]} \\
\left(\mu_{1}, \ldots, \mu_{n}\right)^{*}(f) & =\lambda[M] .\left[\left(Q_{f}\left[\gamma_{1}:=\mu_{1}, \ldots, \gamma_{n}:=\mu_{n}\right]\right) M\right]
\end{aligned}
$$

It is easy to verify that the objects $\Lambda_{s t}, \Lambda_{\sigma \times \tau}$ and $\Lambda_{\sigma \rightarrow \tau}$ provide $H n$ with a semi-ccc structure, and similarly for the second order product.

We shall check that $H 0$ is a $\lambda 1$-model and that $H$ preserves enough points of 1 , in order to establish that a $\lambda 2$-model is obtained (cf. definition 3.2).

In $H 0$ one has $1_{\sigma, \tau}=\lambda[M] \in \Lambda_{s t} .[\lambda x: \sigma \rightarrow \tau \lambda y: \tau . x y]$. Suppose that $f, g: \Lambda_{s t} \rightarrow \Lambda_{\sigma \rightarrow \tau}$ satisfy $\forall x: \Lambda_{s t} \rightarrow \Lambda_{\sigma} . f \cdot x=g \cdot x$ and that $[M] \in \Lambda_{s t}$. A variable $y: \sigma$ gives rise to an arrow $\lambda[N]$. $[y]$ : $\Lambda_{s t} \rightarrow \Lambda_{\sigma}$ and so we have that $\left(Q_{f} M\right) y=\left(Q_{g} M\right) y$. But then $1_{\sigma, \tau} \cdot f=1_{\sigma, \tau} \cdot g$ holds by the $\xi$-rule and thus is the Meyer-Scott axiom satisfied in $H 0$.

Moreover, if $f, g: \Lambda_{\sigma} \rightarrow \Lambda_{\tau}$ in $H 1$ satisfy $\forall \mu: 0 \rightarrow 1 . \mu^{*}(f)=\mu^{*}(g)$, then for $[M] \in \Lambda_{\sigma}$ one has $\mu^{*}(f)\left(\left[M\left[\gamma_{1}:=\mu_{1}\right]\right]\right)=\mu^{*}(g)\left(\left[M\left[\gamma_{1}:=\mu_{1}\right]\right]\right)$ and thus $\left(Q_{f} M\right)\left[\gamma_{1}:=\mu_{1}\right]=$ $\left(Q_{g} M\right)\left[\gamma_{1}:=\mu_{1}\right]$. But then $Q_{f} M=Q_{g} M$, since substitution of (closed) types does not change the reduction possibilities. Hence $f=g$.

It seems reasonable to call this the open term model of $\lambda 2$ and the one from 6.1 the closed term model. Of course, these models can be used to prove familiar completeness results, see Barendregt (1984, 5.2.12).

\section{3 .}

Before describing three ideal models, we mention some (standard) preliminaries. Let $\langle D, \leqslant\rangle$ be a cpo. A subset $I \subseteq D$ is called an ideal in $D$ iff (i) $\perp_{D} \in I$ (or $I \neq \varnothing$ ); (ii) $x \leqslant y \in I \Rightarrow x \in I$; (iii) directed $X \subseteq I \Rightarrow \bigsqcup X \in I$. Ideals are the non-empty closed subsets with respect to the Scott topology. With the ordering inherited from $D$ they form cpos themselves. We write $\mathfrak{I}(D)$ for the collection of ideals in $D$. Since $\mathfrak{I}(D)$ is closed under arbitrary intersection, we may define for any subset $Y \subseteq D$, the ideal generated by $Y$ as $\downarrow Y=\bigcap\{I \in \mathfrak{T}(D) \mid I \supseteq Y\}$. It is the smallest ideal containing $Y$. 
For an arbitrary collection $\left\{I_{\alpha}\right\}$ of ideals, we can take as supremum $\bigsqcup_{\alpha} I_{\alpha}=\downarrow\left(\bigcup_{\alpha} I_{\alpha}\right)$. This makes $\langle\mathfrak{I}(D), \subseteq\rangle$ a complete lattice.

Proposition 6.3.1. $\langle\mathfrak{I}(D), \subseteq\rangle$ is $(\omega-)$ algebraic whenever $\langle D, \leqslant\rangle$ is $(\omega-)$ algebraic. Compact ideals of the form $\downarrow E$ for a finite set $E \subseteq D^{0}$ (the compact elements in $D$ ).

Proof. First show that for a non-empty downward closed subset $Y \subseteq D$ one has $\downarrow Y=\left\{\bigsqcup X \mid X \subseteq Y^{0}\right.$ is directed $\}$; then $\left(\bigsqcup_{\alpha} I_{\alpha}\right)^{0}=\bigcup_{\alpha} I_{\alpha}^{0}$. The rest is standard.

\section{3 .2}

From now on we restrict ourselves to ideals on $P \omega$ (see e.g. Scott, 1976, or Barendregt, 1984) and write $\mathfrak{I}=\mathfrak{I}(P \omega)$. There are three reasons to do so.

(a) There is a bijective (and even effective) pairing $\left\langle\left\langle_{-},-\right\rangle\right\rangle: \omega^{2} \rightarrow \omega$, with projections $p, p^{\prime}$. This gives the possibility to define a continuous function

$$
\dot{U}: P \omega \times P \omega \rightarrow P \omega \quad \text { by } \quad x \dot{U} y=\{\langle\langle n, 0\rangle\rangle \mid n \in x\} \cup\{\langle\langle m, 1\rangle\rangle \mid m \in y\},
$$

which can be used to define a product ideal by

$$
I \times J=\{x \dot{\cup} y \mid x \in I \& y \in J\} .
$$

(b) $P \omega$ is reflexive: there are functions $F: P \omega \rightarrow[P \omega \rightarrow P \omega]$ and $G:[P \omega \rightarrow P \omega] \rightarrow P \omega$ with

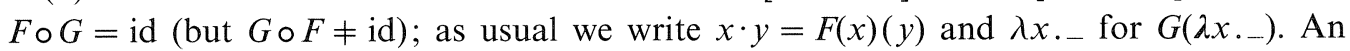
exponent ideal can be defined by $I \rightarrow J=\{x \in D \mid \forall y \in I \cdot x \cdot y \in J\}$. One has $\bigcap_{\alpha} .\left(I_{\alpha} \rightarrow J_{\alpha}\right) \subseteq$ $\left(\bigcap_{\alpha} . I_{\alpha}\right) \rightarrow\left(\bigcap_{\alpha} . J_{\alpha}\right)$ and $\bigcap_{\alpha} .\left(I \rightarrow J_{\alpha}\right)=I \rightarrow\left(\bigcap_{\alpha} . J_{\alpha}\right)$ see the second order type inclusion relations in Mitchell (1988) or Jacobs et al. (1989).

By choosing $\operatorname{Hom}(I, J)=\{f \in[P \omega \rightarrow P \omega] \mid f(I) \subseteq J\}$ one can form a $\lambda 1$-algebra based on I. In order to obtain a $\lambda 1$-model, we put $\operatorname{Hom}(I, J)=[I \rightarrow J]$ - where $I, J$ are considered as cpos - and use the following trick from Martini (1987). For $f \in[I \rightarrow J]$ an extension $f^{+} \in[P \omega \rightarrow P \omega]$ with $\left.f^{+}\right|_{I}=f$ is defined by $f^{+}(z)=\bigsqcup\left\{f(e) \mid e \in I^{0}\right.$ and $\left.e \leqslant z\right\}$, using that $P \omega$ is a complete lattice. Then we write $\lambda z: I . f(z)=G\left(f^{+}\right)$which is an element of $I \rightarrow J$.

(c) There is a continuous injection $\mathbf{i}: \mathfrak{I} \rightarrow P \omega$, given by $\mathbf{i}(I)=\left\{n \in \omega \mid e_{n} \in I^{0}\right\}$, where $e_{n} \in P \omega^{0}$ are finite sets (with codenumber $n$ ). Since ideals are determined by their compact elements (i.e. $I=\downarrow I^{0}$ ), this function $\mathbf{i}$ is an injection. It is also continuous, since for a directed collection $\left\{I_{\alpha}\right\}$ in $\mathfrak{I}$ one has $\mathbf{i}\left(\bigsqcup_{\alpha} I_{\alpha}\right)=\left\{n \mid e_{n} \in\left(\sqcup_{\alpha} I_{\alpha}\right)^{0}\right\}=\left\{n \mid e_{n} \in \bigcup_{\alpha} I_{\alpha}^{0}\right\}=\bigcup_{\alpha}$ $\left\{n \mid e_{n} \in I_{\alpha}^{0}\right\}=\bigcup_{\alpha} \mathbf{i}\left(I_{\alpha}\right)$. This injection also preserves compactness and one even has $I \subseteq J \Leftrightarrow \mathbf{i}(I)$ $\subseteq \mathbf{i}(J)$. A continuous application map $\cdot: P \omega \times \mathfrak{I} \rightarrow P \omega$ can now be defined by $x \cdot I=x \cdot \mathbf{i}(I)$.

Again following Martini (1987), we define for $f \in[\mathfrak{I} \rightarrow P \omega]$ a function $f^{++} \in[P \omega \rightarrow P \omega]$ with $f=f^{++}$o i by $f^{++}(z)=\bigcup_{E}\{f(\downarrow E) \mid \mathbf{i}(\downarrow E) \subseteq z\}$, where $E$ ranges over finite subsets of $D^{0}$. Writing $\lambda I: \mathfrak{I} . f(I)=G\left(f^{++}\right)$, we get $(\lambda I: \mathfrak{I} . f(I)) \cdot J=f^{++}(\mathbf{i}(J))=f(J)$; this is beginning to look like second order $\lambda$-calculus.

6.4 .

The first ideal $\lambda 2$-algebra we describe comes from MacQueen et al. (1986). The base category $\mathbf{B}$ has objects $n \in \omega$ (as in 6.1) and arrows $u=\left(u_{1}, \ldots, u_{n}\right): m \rightarrow n$ are given by maps $u_{i}: \mathfrak{I}^{m} \rightarrow \mathfrak{I}$. As before, $n+m$ forms a product, 0 is terminal and $\Omega=1$. 
The functor $H: \mathbf{B}^{\text {op }} \rightarrow \mathbf{C a t}$ has fibres $H n$ with arrows $X: n \rightarrow 1$ from $\mathbf{B}$ as objects; morphisms $f: X \rightarrow Y$ in $H n$ are functions $f \in[P \omega \rightarrow P \omega]$ with $f\left(X_{\mathrm{I}}\right) \subseteq Y_{\mathrm{I}}$ for all $\mathbb{I} \in \mathfrak{I}^{n}$, where we consider $X$ as a family $\left\{X_{\mathbf{I}}\right\}$ with $X_{\mathbf{I}}=X(\boldsymbol{I})$. For $u: m \rightarrow n$ in $\mathbf{B}$, we have $u^{*}: H n \rightarrow H m$ given by $u^{*}(X)=X \circ u$ and $u^{*}(f)=f$.

The semi-ccc structure of $H n$ is given by a terminal $t_{n}=\lambda \mathbb{I} \in \mathfrak{I}^{n} .\{\varnothing\}$ and semi-product $X \times Y=\lambda \mathbf{I} \in \mathfrak{T}^{n} . X_{\mathbf{I}} \times Y_{\mathbf{I}}$ with projection $\pi=\lambda x \in P \omega .\{n \in \omega \mid\langle\langle n, 0\rangle\rangle \in x\}: X \times Y \rightarrow X$; for $f$ : $Z \rightarrow X$ and $g: Z \rightarrow Y$, one has $\langle f, g\rangle=\lambda x \in P \omega . f(x) \dot{\cup} g(x): Z \rightarrow X \times Y$. The semi-exponent $Y^{X}=\lambda \mathbb{I} \in \mathfrak{I}^{n} . X_{\mathbf{I}} \rightarrow Y_{\mathbf{I}}$ has $e v=\lambda x \in P \omega \cdot \pi(x) \cdot \pi^{\prime}(x)$ and for $f: Z \times X \rightarrow Y$ an exponential adjunct $\Lambda(f)=\lambda x \in P \omega . \lambda y . f(x \dot{\cup} y): Z \rightarrow Y^{X}$.

Product functors $\Pi_{n}: H(n+1) \rightarrow H n$ are given by $\Pi_{n}(Y)=\lambda \mathbf{I} \in \mathfrak{I}^{n} . \cap_{J} . Y_{\mathbf{I}, J}$ and $\Pi_{n}(f)=f$. The adjunction $\pi_{n, 1} \dashv \Pi_{n}$ is then a triviality.

Analogously one can define sum functors by $\Sigma_{n}(Y)=\lambda \mathbb{I} \in \mathfrak{I}^{n} \cdot \bigsqcup_{J} . Y_{\mathbf{I}, J}$ and $\Sigma_{n}(f)=f$.

6.5 .

The second ideal $\lambda 2$-algebra - which is a simple version of a construction used in Martini (1987) - has non-extensional type abstraction, i.e. semi-functors for the second order product. The base category and the objects of the fibres are as before. As to the arrows in the fibres, we now put $f: X \rightarrow Y$ in $H n \Leftrightarrow f \in\left[\mathfrak{I}^{n} \times P \omega \rightarrow P \omega\right]$ such that $f_{\mathrm{I}}\left(X_{\mathrm{I}}\right) \subseteq Y_{\mathrm{I}}$ for all $\mathbf{I} \in \mathfrak{I}^{n}$, where $f_{\mathbf{I}}(x)$ is written for $f(\mathbb{I}, x)$. The composition is given by $g \circ f=\lambda \mathbb{I} \in \mathfrak{I}^{n} \cdot g_{\mathbf{I}} \circ f_{\mathbf{I}}$ and $u^{*}(f)=f \circ u$. The semi-ccc structure of the fibres is virtually as before, e.g. now $\Lambda(f)=\lambda \mathbb{I} \in \mathfrak{T}^{n} \lambda x \in P \omega . \lambda y . f_{\mathbf{I}}(x \dot{\cup} y)$. The major change concerns the product semifunctors $\Pi_{n}$,

$$
\begin{gathered}
Y \mapsto \lambda \mathbb{I} \in \mathfrak{I}^{n} \cdot\left\{x \in P \omega \mid \forall J \in \mathfrak{T} . x \cdot J \in Y_{\mathbf{I}, J}\right\} \\
f \mapsto \lambda \mathbb{I} \in \mathfrak{I}^{n} \lambda x \in P \omega . \lambda J: \mathfrak{I} \cdot f_{\mathbf{I}, J}(x \cdot J),
\end{gathered}
$$

see 6.3.2(c). The semi-adjunction

$$
\begin{aligned}
& H(N+1)\left(\pi_{n, 1}^{*}(X), Y\right) \rightleftarrows H(n)\left(X, \Pi_{n}(Y)\right) \quad \text { is given by } \\
& f \mapsto \lambda \mathbb{I} \in \mathfrak{T}^{n} \lambda x \in P \omega . \lambda J: \mathfrak{T} \cdot f_{\mathbf{I}, J}(x) \\
& \lambda \mathbb{I}, J \in \mathfrak{I}^{n+1} \lambda x \in P \omega \cdot g_{\mathbf{I}}(x) \cdot J \leftarrow g .
\end{aligned}
$$

6.6.

The ideal $\lambda 2$-algebra that we started with in 6.4 can be transformed into a $\lambda 2$-model by the following construction - which can be considered as a 'non-extensional collaps', a bit like in the PER-model in 6.7.

Given ideals $I, J$ we define an equivalence relation $\sim$ one $I \rightarrow J=\{x \in P \omega \mid \forall y \in I . x \cdot y \in J\}$ by $x_{1} \sim x_{2} \Leftrightarrow \forall y \in I . x_{1} \cdot y=x_{2} \cdot y$ and put $I \Rightarrow J=I \rightarrow J / \sim$. Elements of $I \Rightarrow J$ are written as $[x]_{I \rightarrow J}$. Notice that $[x]_{I \rightarrow J}=[\lambda y . x \cdot y]_{I \rightarrow J}$.

The changes (with respect to 6.4) only concern the morphisms in the fibres. We now define $f: X \rightarrow Y$ in $H n \Leftrightarrow f$ is a family of equivalence classes $f_{\mathbf{I}} \in X_{\mathbf{I}} \Rightarrow Y_{\mathbf{I}}$ which has a realiser, i.e. $\exists z \in P \omega \forall \mathbf{I} \in \mathfrak{I}^{n} \cdot f_{\mathbf{I}}=[z]_{X_{\mathbf{I}} \rightarrow Y_{\mathbf{I}}}$. 
Then $\operatorname{id}_{X}=\lambda \mathbf{I} \in \mathfrak{I}^{n} \cdot[\lambda x \cdot x]_{X_{\mathbf{I}} \rightarrow X_{\mathbf{I}}}$ and for $g: Y \rightarrow Z$ one has $g \circ f=\lambda \mathbf{I} \in \mathfrak{I}^{n} \cdot\left[\lambda x \cdot z_{g}\right.$. $\left.\left(z_{f} \cdot x\right)\right]_{X_{1} \rightarrow z_{1}}$.

The arrow part of the semi-ccc structure on $\mathrm{Hn}$ is given as follows.

Terminal

Product

$$
\begin{aligned}
!_{X} & =\lambda \mathbf{I} \in \mathfrak{T}^{n} \cdot[\lambda x \cdot \varnothing]_{X_{\mathbf{I}} \rightarrow\left(t_{n}\right)_{\mathbf{I}}} \\
\pi & =\lambda \mathbf{I} \in \mathfrak{I}^{n} \cdot[\lambda x \cdot\{n \mid\langle\langle n, 0\rangle\rangle \in x\}]_{(X \times Y)_{\mathbf{I}} \rightarrow X_{\mathbf{I}}}, \\
\langle f, g\rangle & =\lambda \mathbf{I} \in \mathfrak{I}^{n} \cdot\left[\lambda x \cdot z_{f} \cdot x \dot{\mathrm{U}} z_{g} \cdot x\right]_{Z_{\mathbf{I}} \rightarrow(X \times Y)_{\mathbf{I}}}
\end{aligned}
$$

Semi-exponent

$$
\begin{aligned}
\Lambda(f) & =\lambda \mathbf{I} \in \mathfrak{I}^{n} \cdot\left[\lambda x y \cdot z_{f} \cdot(x \dot{U} y)\right]_{Z_{\mathbf{I}} \rightarrow\left(Y^{X}\right)_{\mathbf{I}}}, \\
e v & =\lambda \mathbf{I} \in \mathfrak{T}^{n} \cdot[\lambda x \cdot\{n \mid\langle\langle n, 0\rangle\rangle \in x\} \cdot\{n \mid\langle\langle n, 1\rangle\rangle \in x\}]_{\left(Y^{X} \times X\right)_{\mathbf{I}} \rightarrow Y_{\mathbf{I}}} .
\end{aligned}
$$

One has

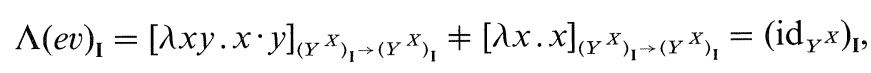

since otherwise $\lambda x y \cdot x \cdot y \sim \lambda x \cdot x$ in $\left(Y^{X}\right)_{\mathbf{I}} \rightarrow\left(Y^{X}\right)_{\mathbf{I}}$ and thus for all $x \in\left(Y^{X}\right)_{\mathbf{I}}, \lambda y \cdot x \cdot y=x$. But the latter does not hold in general in $P \omega$. Hence the fibre categories are semi-ccc's and we do not have a model with extensional (term) abstraction. The reason for this lies in the fact that we have used different arrows $\Rightarrow$ and $\rightarrow$ for homsets resp. exponents, cf. the PER-model in the next section.

The arrow part of the second order product is given by

$$
\Pi_{n}(f: Y \rightarrow Z)=\lambda \mathbf{I} \in \mathfrak{I}^{n} \cdot\left[z_{f}\right]_{\cap_{J} . Y_{\mathbf{I}, J} \rightarrow \cap_{J} . Z_{\mathbf{I}, J}},
$$

which gives an adjunction $\pi_{n, 1} \dashv \Pi_{n}$.

It is easy to see that $H$ preserves enough points of 1 , and so we only have to check that $H 0$ is a $\lambda 1$-model in order to conclude that we have constructed a $\lambda 2$-model. Since $t_{n}=\lambda \mathbf{I} \in \mathfrak{I}^{n} .\{\phi\}$ is terminal in $H n$, we use proposition 2.2.2(2). Suppose $f, g: X \rightarrow Y$ in $H 0$ satisfy $\forall x: t_{0} \rightarrow X . f \circ x=g \circ x$. Then for all $y \in X_{\varnothing}, \lambda x . z_{f} \cdot y \sim \lambda x \cdot z_{g} \cdot y$ in $\{\varnothing\} \rightarrow Y_{\varnothing}$. Hence $z_{f} \cdot y=z_{g} \cdot y$ and thus $z_{f} \sim z_{g}$ in $X_{\varnothing} \rightarrow Y_{\varnothing}$. Hence $f=g$.

\section{7 .}

In order to give an example of a $\lambda \eta$ 2-model, we describe the PER-model (due to Kreisel, Girard, Troelstra and Moggi) in the framework of this paper. The starting point is Kleene's partial applicative structure $\langle\omega, \cdot\rangle$ given by $n \cdot m=\phi_{n}(m)$, where $\phi_{n}$ denotes the $n$th partial recursive function. Whenever such an application $n \cdot m$ is written, it is understood that $m \in \operatorname{dom} \phi_{n}$. If $f$ is partial recursive and $e$ its index or codenumber (i.e. $f=\phi_{e}$ ), one often writes $\lambda x . f(x)$ for $e$; then $(\lambda x . f(x)) \cdot y=f(y)$. Using the $s-m-n$ theorem, one can also work with codenumbers like $\lambda x y \cdot g(x, y)$ - with obvious meaning.

A partial equivalence relation (on $\omega$ ) is a subset $R \subseteq \omega \times \omega$ which is both symmetric and transitive. PER denotes the set of all such relations; it is closed under arbitrary intersections and so we can put $\downarrow A=\bigcap\{R \in \mathrm{PER} \mid R \supseteq A\}$ for the smallest p.e.r containing an arbitrary 
$A \subseteq \omega \times \omega$. If $A$ is symmetric, this amounts to taking the transitive closure of $A$. PER is also closed under $\times, \rightarrow$ and $\bigsqcup$ in the following way.

$$
\begin{aligned}
R \times S & =\left\{(n, m) \mid p n R p m \& p^{\prime} n S p^{\prime} n\right\} \quad \text { with } p, p^{\prime} \text { as in } 6.3 .2 \\
R \rightarrow S & =\{(n, m) \mid \forall k, l . k R l \Rightarrow n \cdot k S m \cdot l\} \\
\bigsqcup_{\alpha} \cdot R_{\alpha} & =\downarrow\left(\bigcup_{\alpha} \cdot R_{\alpha}\right) .
\end{aligned}
$$

For $R \in$ PER we write $\operatorname{dom} R=\{n \in \omega \mid n R n\}$ and $Q(R)=\left\{[n]_{R} \mid n \in \operatorname{dom} R\right\}$, where $[n]_{R}=$ $\{m \in \omega \mid m R n\}$. Notice that $\bigcap_{\alpha} \operatorname{dom}\left(R_{\alpha} \rightarrow S_{\alpha}\right) \subseteq \operatorname{dom}\left(\bigcap_{\alpha} R_{\alpha} \rightarrow \bigcap_{\alpha} S_{\alpha}\right)$ and $\bigcap_{\alpha} \operatorname{dom}\left(R \rightarrow S_{\alpha}\right)=$ $\operatorname{dom}\left(R \rightarrow \bigcap_{\alpha} S_{\alpha}\right)$. The latter equality gives the adjunction for the second order product, as will be shown below. Analogously, one has $\bigcap_{\alpha} \operatorname{dom}\left(R_{\alpha} \rightarrow S_{\alpha}\right) \subseteq \operatorname{dom}\left(\bigsqcup_{\alpha} R_{\alpha} \rightarrow \bigsqcup_{\alpha} S_{\alpha}\right)$ and $\bigcap_{\alpha} \operatorname{dom}\left(R \rightarrow S_{\alpha}\right)=\operatorname{dom}\left(\bigsqcup_{\alpha} R_{\alpha} \rightarrow S\right)$, which is the basis for second order sums.

The base category $\mathbf{B}$ is as before, but this time arrows $u=\left(u_{1}, \ldots, u_{n}\right): m \rightarrow n$ have components $u_{i}: \mathrm{PER}^{m} \rightarrow \mathrm{PER}$. The functor $H: \mathbf{B}^{\text {op }} \rightarrow$ Cat is given as follows. Objects of $H n$ are morphisms $X: n \rightarrow 1$ in $\mathbf{B}$ and arrows $f: X \rightarrow Y$ in $H n$ are families of equivalence classes $f_{\mathbf{R}} \in Q\left(X_{\mathbf{R}} \rightarrow Y_{\mathbf{R}}\right)$ which have a realiser $n$ such that $\forall \mathbf{R} \in \mathrm{PER}^{n} \cdot f_{\mathbf{R}}=[n]_{X_{\mathbf{R}} \rightarrow Y_{\mathbf{R}}}$. Similarly to 6.6 one has $\operatorname{id}_{X}=\lambda \mathbf{R} \in \mathrm{PER}^{n} \cdot[\lambda x . x]_{X_{\mathbf{R}} \rightarrow X_{\mathbf{R}}}$ and $g \circ f=\lambda \mathbf{R} \in \operatorname{PER}^{n} \cdot\left[\lambda x \cdot n_{g} \cdot\left(n_{f} \cdot x\right)\right]_{X_{\mathbf{R}} \rightarrow Z_{\mathbf{R}}} \cdot$

The fibres $H n$ have a ccc-structure with terminal $t_{\mathrm{n}}=\lambda \mathbf{R}$. $\{(0,0)\}$, product $X \times Y=$ $\lambda \mathbb{R} . X_{\mathbf{R}} \times Y_{\mathbf{R}}$ and exponent $Y^{X}=\lambda \mathbf{R} . X_{\mathbf{R}} \rightarrow Y_{\mathbf{R}}$. The functors $\Pi_{n}: P H(n+1) \rightarrow H n$ are given as follows.

$$
\begin{gathered}
Y \mapsto \lambda \mathbf{R} \in \mathrm{PER}^{n} \cdot \bigcap_{S \in \mathrm{PER}} \cdot Y_{\mathbf{R}, S} \\
f: Y \rightarrow Z \mapsto \lambda \mathbf{R} \in \mathrm{PER}^{n} \cdot\left[n_{f}\right]_{\cap_{s} \cdot Y_{\mathbf{R}, S} \rightarrow \cap_{S} \cdot Z_{\mathbf{R}, S}}
\end{gathered}
$$

It is easy to show that this determines a $\lambda \eta 2$-model. It also admits sums by the following definition of $\Sigma_{n}: H(n+1) \rightarrow H n$.

$$
\begin{gathered}
Y \mapsto \lambda \mathbf{R} \in \mathbf{P E R}^{n} \cdot \bigsqcup_{S \in \mathbf{P E R}} \cdot Y_{\mathbf{R}, S} \\
f: Y \rightarrow Z \mapsto \lambda \mathbb{R} \in \mathrm{PER}^{n} \cdot\left[n_{f}\right]_{\bigsqcup_{s} \cdot Y_{\mathbf{R}, S} \rightarrow \bigsqcup_{S} \cdot Z_{\mathbf{R}, S}}
\end{gathered}
$$

Above we have described the 'core' of the PER-construction; in relation with the categories $\omega$-Sets or $\mathbb{E f f}$, models of more complicated calculi can be obtained, see e.g. Longo and Moggi (1987), Hyland (1989), Ehrhard (1988) or Streicher (1988). Other $\lambda \eta 2-$ models are the closure models of $P \omega$ (Scott, 1976; Barendregt and Rezus, 1983; Seely, 1987; or Bruce et al., 1990), the qualitative/coherence model (Girard, 1986; Girard et al., 1989) or the domain theoretic model (Coquand et al., 1989).

\section{Bruce-Meyer-Mitchell-Models}

In this section we start by reviewing the BMM-model definition. The next step is to collect these models in a suitable category, which makes it possible to relate them in theorems 7.3 and 7.4.3 to the previously defined categorical models.

7.1 .

In Bruce and Mayer (1984) and Bruce et al. (1990) a notion of the model for $\lambda 2$ is introduced. The construction roughly consists of a hierarchy of sets (as for first order $\lambda$ - 
calculus models, see Friedman, 1975) with collections of functions between them (as for reflexive domains). The domains of the abstraction functions are not completely specified but are required to be such that they contain the $\lambda 2$-polynomials (and analogously for the $\Delta$-function used to interpret universal types). In this way the model definition explicitly depends on the syntax, see under 7.1.2 for a more detailed discussion.

In order to fix the notation, we briefly recall the definition of a BMM-model.

A type domain $\mathbf{T}$ is a tuple $\langle T, \backsim, \Delta\rangle$, where $T$ is a set of so-called type representatives, $\leadsto$ is a function $T \times T \rightarrow T$ and $\Delta$ a (partial) function $T^{T} \rightarrow T$. The domain of $\Delta$ is denoted by $[T \rightarrow T]$ and should be such that types can be interpreted in $\mathbf{T}$ as follows. Given a type valuation $\xi$ : Typevar $\rightarrow T$ one has

$$
\begin{aligned}
\llbracket \gamma \rrbracket_{\xi} & =\xi(\gamma) \\
\llbracket \sigma \rightarrow \tau \rrbracket_{\xi} & =\llbracket \sigma \rrbracket_{\xi} \leadsto \llbracket \tau \rrbracket_{\xi} \\
\llbracket \forall \gamma: * . \sigma \rrbracket_{\xi} & =\Delta\left(\lambda a \in T . \llbracket \sigma \rrbracket_{\xi(\gamma:=\alpha)}\right) .
\end{aligned}
$$

Notice that one can immediately prove $\llbracket \sigma[\gamma:=\tau] \rrbracket_{\xi}=\llbracket \sigma \rrbracket_{\xi\left(\gamma:=[\tau]_{\xi}\right)}$ and similarly for simultaneous substitution.

A second order functional domain is a tuple $\mathbf{m}=\left\langle\mathbf{T},\left\{D_{a}\right\}_{a \in T},\left\{F_{a b}, G_{a b}\right\}_{a, b \in T},\left\{F_{f}, G_{f}\right\}_{f \in[T \rightarrow T]}\right\rangle$ where the $D$ 's are sets and the $F$ and $G$ 's are functions between them in the following way.

$$
F_{a b}: D_{a \rightsquigarrow b} \rightarrow\left[D_{a} \rightarrow D_{b}\right] \quad G_{a b}:\left[D_{a} \rightarrow D_{b}\right] \rightarrow D_{a \rightsquigarrow b},
$$

satisfying $F_{a b} \circ G_{a b}=\mathrm{id}_{\left[D_{a} \rightarrow D_{b}\right]}$, for a certain set $\left[D_{a} \rightarrow D_{b}\right] \subseteq D_{b}^{D_{a}}$.

$$
F_{f}: D_{\Delta(f)} \rightarrow\left[\Pi_{a \in T} . D_{f(a)}\right] \quad G_{f}:\left[\Pi_{a \in T} . D_{f(a)}\right] \rightarrow D_{\Delta(f)},
$$

satisfying $F_{f} \circ G_{f}=\mathrm{id}_{\left[\Pi_{a \in T} . D_{f(a)}\right]}$, for a certain set $\left[\Pi_{a \in T} . D_{f(a)}\right] \subseteq \Pi_{a \in T} . D_{f(a)}$.

The notation $F_{a b}, G_{a b}, F_{f}$ and $G_{f}$ is a bit cumbersome; we shall often write

$$
\begin{array}{rll}
x \cdot y & \text { for } & F_{a b}(x)(y) \text { if } x \in D_{a \rightsquigarrow b} \text { and } y \in D_{a} \\
\lambda x: a \cdot t(x) & \text { for } & G_{a b}(t) \text { if } t \in\left[D_{a} \rightarrow D_{b}\right] \\
x \cdot a & \text { for } & F_{f}(x)(a) \text { if } x \in D_{\Delta(f)} \\
\lambda a: T . s(a) & \text { for } & G_{f}(s) \text { if } s \in\left[\Pi_{a \in T} . D_{f(a)}\right] .
\end{array}
$$

Then $(\lambda x: a . t(x)) \cdot y=t(y)$ and $(\lambda a: T . s(a)) \cdot b=s(b)$.

Such a second order functional domain $\mathbf{m}$ is called a second order model or better a $B M M$-model if terms can be interpreted as follows. For a term $\Gamma+\Theta \vdash M: \sigma$ together with a type valuation $\xi$ :Typevar $\rightarrow T$ and a term valuation $\rho$ :Termvar $\rightarrow \bigcup_{a \in T} . D_{a}$ such that $\xi, \rho \models \Gamma+\Theta$ - i.e. $\rho(y) \in D_{[\tau]_{\xi}}$ for $y: \tau \in \Theta-$ one has

$$
\begin{aligned}
\llbracket x \rrbracket_{\xi+\rho} & =\rho(x) \\
\llbracket M N \rrbracket_{\xi+\rho} & =\llbracket M \rrbracket_{\xi+\rho} \cdot \llbracket N \rrbracket_{\xi+\rho} \\
\llbracket \lambda x: \sigma \cdot M \rrbracket_{\xi+\rho} & =\lambda z: \llbracket \sigma \rrbracket_{\xi} \cdot \llbracket M \rrbracket_{\xi+\rho(x:=z)} \\
\llbracket M \tau \rrbracket_{\xi+\rho} & =\llbracket M \rrbracket_{\xi+\rho} \cdot \llbracket \tau \rrbracket_{\xi} \\
\llbracket \lambda \gamma: * . M \rrbracket_{\xi+\rho} & =\lambda a: T \cdot \llbracket M \rrbracket_{\xi(\gamma:=a)+\rho} .
\end{aligned}
$$


As for type interpretation, a number of substitution lemmas can now be proved. In the sequel we always suppose that $\xi$ and $\rho$ validate the appropriate contexts whenever we write $\llbracket \sigma \rrbracket_{\xi}$ or $\llbracket M \rrbracket_{\xi+\rho}$.

7.1.1.

Given such a BMM-model $\mathbf{m}$, one can define a calculus $2 \lambda(\mathbf{m})$ extended with type and term constants from $\mathbf{m}$ as in 4.5 for $\lambda 2$-algebras. For a type representative $a \in T$ one adds a type constant $c_{a}$ with $\vdash c_{a}: *$ and $\llbracket c_{a} \rrbracket_{\xi}=a$; for a closed type $\sigma$ and an element $d \in D_{\llbracket \sigma]}$ one adds a term constant $c_{d}^{\sigma}$ with $\vdash c_{d}^{\sigma}: \sigma$ and $\llbracket c_{d}^{\sigma} \rrbracket_{\xi+\rho}=d$.

7.1.2.

Let us explicitly state the conditions which have to be satisfied in order that a functional domain yields a BMM-model, see the last definition of section 3 in Bruce and Meyer (1984) and the preceding alinea.

(1) For every type $\sigma$ and valuation $\xi, \lambda a \in T . \llbracket \sigma \rrbracket_{\xi(\gamma:=a)} \in[T \rightarrow T]$.

For every pair of corresponding valuation functions $\xi, \rho$ and every term $M$,

(2) if $M(x): \tau$ for $x: \sigma$, then $\lambda z \in D_{\llbracket \sigma \rrbracket_{\xi}} \cdot \llbracket M \rrbracket_{\xi+\rho(x:=z)} \in\left[D_{\llbracket \sigma \rrbracket_{\xi}} \rightarrow D_{\llbracket \tau \rrbracket_{\S}}\right]$;

(3) if $M(\gamma): \sigma(\gamma)$ - with $\gamma$ not free in the types of free term variables in $M$ - then

$$
\lambda a \in T . \llbracket M \rrbracket_{\xi(\gamma ;=a)+\rho} \in\left[\Pi_{a \in T} . D_{\llbracket \sigma \rrbracket_{\xi(\gamma ;=a)}}\right] .
$$

These syntactical conditions are not very elegant: if we distinguish a syntactical from a semantical level, we would surely want a notion of model to live at the semantical level. And indeed, the (categorical) notions from section 3 are purely semantical, but the BMMmodels as presented above seem to hang somewhere halfway between syntax and semantics.

In the semantics of the untyped $\lambda$-calculus (see Barendregt, 1984, ch. 5) we find a similar situation. There are purely semantical notions, with combinators or with a reflexive object in a ccc. But there is also the Hindley-Longo notion of a model, which consists of a set with application and an interpretation map satisfying syntactical conditions. An important result is that these notions are all equivalent (in a certain sense), which gives a semantical justification for the syntactical conditions in Hindley-Longo models.

In the second order case, such a semantical justification is given in Bruce et al. (1990, section 6) using second order combinators. That paper deals essentially with extensional BMM-models (where $G_{a b} \circ F_{a b}=$ id and $G_{f} \circ F_{f}=\mathrm{id}$ ). Non-extensional ones are briefly discussed in section 6.6.

These syntactical conditions will play an important role in the rest of this section. In fact, when dealing with a BMM-model $\mathbf{m}=\langle T, \ldots\rangle$, we shall consider the sets $[T \rightarrow T]$, $\left[D_{a} \rightarrow D_{b}\right]$ and $\left[\Pi_{a \in T} . D_{f(a)}\right]$ to be minimal, i.e. to contain only the type and term polynomials as described in the beginning of 7.1.2 above.

Definition 7.2. In order to obtain a category $\mathbf{B M M}$, let $\mathbf{m}=\langle T, \ldots\rangle$ and $\mathbf{m}^{\prime}=\left\langle T^{\prime}, \ldots\right\rangle$ be BMM-models; an arrow $\mathbf{m} \rightarrow \mathbf{m}^{\prime}$ consists of a pair $\langle\psi,\{\chi\}\rangle$ with $\psi: T \rightarrow T^{\prime}$ and $\chi_{a}: D_{a} \rightarrow D_{\psi(a)}^{\prime}$ such that for $\sigma, M$ from $\lambda 2(\mathbf{m})$ one has

$$
\begin{aligned}
\psi\left(\llbracket \sigma \rrbracket_{\xi}^{\mathrm{m}}\right) & =\llbracket \sigma_{\psi} \rrbracket_{\psi \circ \xi}^{\mathrm{m}^{\prime}} \\
\chi\left(\llbracket M \rrbracket_{\xi+\rho}^{\mathrm{m}}\right) & =\llbracket M_{\psi, \chi} \rrbracket_{\psi \circ \xi+\chi \circ \rho}^{\mathbf{m}^{\prime}}
\end{aligned}
$$


where $\sigma_{\psi}$ and $M_{\psi, \chi}$ are obtained from $\sigma, M$ by replacing constants, see lemma 4.5.1. Moreover it is required that

$$
\forall \xi \cdot \llbracket \sigma \rrbracket_{\xi}^{\mathbf{m}}=\llbracket \tau \rrbracket_{\xi}^{\mathbf{m}} \Rightarrow \forall \xi \cdot \llbracket \sigma_{\psi} \rrbracket_{\xi}^{\mathbf{m}^{\prime}}=\llbracket \tau_{\psi} \rrbracket_{\xi}^{\mathrm{m}^{\prime}},
$$

i.e. at least the type equalities from $\mathbf{m}$ are satisfied in $\mathbf{m}^{\prime}$. It is left to the reader to verify that the same can be derived from term equalities (see the proof of lemma 4.5.3).

Theorem 7.3. There are functors $K: \lambda 2-\mathbf{M O D}_{\mathrm{s}} \rightarrow \mathbf{B M M}$ and $L: \mathbf{B M M} \rightarrow \lambda 2-\mathbf{M O D}_{\mathrm{s}}$ with $k \circ L \cong \mathrm{id}$.

Proof. This will occupy the next few pages. We start with $K: \lambda 2-\mathbf{M O D}_{\mathrm{s}} \rightarrow \mathbf{B M M}$.

(a) $K$ on objects. Suppose $H \in \mathbf{2}-\mathbf{M O D}_{\mathrm{s}}$ is as described in section 3. Since one can interpret the calculus $\lambda 2$ in $H$-see section 4 -all we have to do is pick out the relevant parts to obtain a BMM-model $K H$. So we start by taking

$$
T=\mathbf{B}(t, \Omega)
$$

and for $a \in T$, i.e. $a: t \rightarrow \Omega$ in $\mathbf{B}, \quad D_{a}=H t\left(t, \phi_{t}(a)\right)$

which are all (small) sets, since $H$ is small, see definition 3.4.

The BMM-model $K H$ that we are forming will be such that

$$
\llbracket \sigma \rrbracket_{\xi}^{K H}=\llbracket \sigma \rrbracket_{\xi}^{H} \quad \llbracket M \rrbracket_{\xi+\rho}^{K H}=\llbracket M \rrbracket_{\xi+\rho}^{H} .
$$

Hence the following definitions (in which the superscripts $K H$ or $H$ are omitted). For $a, b \in T$, put

$$
a \leadsto b=\phi_{t}^{-1}\left(\phi_{t}(b)^{\phi_{t}(a)}\right)
$$

and for $f=\lambda a \in T . \llbracket \sigma \rrbracket_{\xi(\gamma:=a)} \in[T \rightarrow T]$

$$
\Delta(f)=\llbracket \forall \gamma: * . \sigma \rrbracket_{\xi} .
$$

Because $H$ is small, the object $\Omega \cong t \times \Omega$ has enough points and thus we have that $\Delta$ is welldefined: equal inputs yield equal outputs.

For $x \in D_{a \rightsquigarrow b}$ and $y \in D_{a}$, i.e. $x: t \rightarrow \phi_{t}(b)^{\phi_{t}(a)}$ and $y: t \rightarrow \phi_{t}(a)$ in $H t$, we define

$$
F_{a b}(x)(y)=e v \circ\langle x, y\rangle \in D_{b},
$$

and for $g=\lambda z \in D_{\llbracket \sigma]_{\xi}} \llbracket \llbracket M \rrbracket_{\xi+\rho(x:=z)} \in\left[D_{\llbracket \sigma]_{\xi}} \rightarrow D_{\llbracket \tau]_{\xi}}\right]$,

$$
G_{\llbracket \sigma \rrbracket_{\xi}\left[\tau \rrbracket_{\xi}\right.}(g)=\llbracket \lambda x: \sigma \cdot M \rrbracket_{\xi+\rho},
$$

which is well-defined by theorem 4.4.1.

Suppose $f=\lambda a \in T . \llbracket \sigma \rrbracket_{\xi(\gamma ;=a)} \in[T \rightarrow T]$ and $x \in D_{\Delta}(f)$; the latter implies that $x: t \rightarrow \Pi_{t}\left(\phi_{t \times \Omega}\left(\llbracket \sigma \rrbracket_{\Gamma, \gamma: *} \circ \xi^{\Gamma} \times \mathrm{id}\right)\right)$ in $H t$ and so we put

$$
F_{f}(x)(b)=\left\langle\mathrm{id}_{t}, b\right\rangle^{*}(\beta(t)(x)) \in D_{f(b)},
$$

and for $h=\lambda a \in T . \llbracket M \rrbracket_{\xi(\gamma:=a)+\rho} \in\left[\prod_{a \in T} . D_{\llbracket \sigma] \xi(\gamma:=a)}\right]$

$$
G_{f}(h)=\llbracket \lambda \gamma: * . M \rrbracket_{\xi+\rho},
$$

which is well-defined by theorem 4.4.1 again. 
(b) $K$ on arrows $\langle R, S\rangle: H \rightarrow H^{\prime}$ in $\lambda \mathbf{2}-\mathbf{M O D}_{\mathrm{s}}$. Let us write the BMM-models defined above as $K H=\langle T, \ldots\rangle$ and $K H^{\prime}=\left\langle T^{\prime}, \ldots\right\rangle$. We define $K(\langle R, S\rangle)=\langle\psi,\{\chi\}\rangle: K H \rightarrow K H^{\prime}$ in $\mathbf{B M M}$ by

$$
\begin{gathered}
\psi: T \rightarrow T^{\prime} \quad \text { by } \quad \psi(a)=R a \\
\chi_{a}: D_{a} \rightarrow D_{\psi(a)}^{\prime} \quad \text { by } \quad \chi_{a}(x)=S_{t} x .
\end{gathered}
$$

Then

$$
\begin{aligned}
\psi\left(\llbracket \sigma \rrbracket_{\sigma}^{K H}\right) & =R\left\{\llbracket \sigma \rrbracket_{\Gamma}^{H} \circ\left\langle\mathrm{id}, \xi\left(\gamma_{1}\right), \ldots, \xi\left(\gamma_{n}\right)\right\rangle\right\} \\
& =\llbracket \sigma_{R} \rrbracket_{\Gamma}^{H^{\prime}} \circ\left\langle\mathrm{id}, R \xi\left(\gamma_{1}\right), \ldots, R \xi\left(\gamma_{n}\right)\right\rangle,
\end{aligned}
$$

by lemma $4.5 .1(1)$ and the fact that $R$ preserves the cc structure.

$$
\begin{aligned}
& =\llbracket \sigma_{\psi} \rrbracket_{\Gamma}^{H^{\prime} \circ(\psi \circ \xi)^{\Gamma}} \\
& =\llbracket \sigma_{\psi} \rrbracket_{\psi \circ \xi}^{K H^{\prime}} .
\end{aligned}
$$

Analogously, one can check that $\chi\left(\llbracket M \rrbracket_{\xi+\rho}^{K H}\right)=\llbracket M_{\psi, \chi} \rrbracket_{\psi \circ \xi+\chi \circ \rho}^{K H^{\prime}}$. The last requirement $\forall \xi . \llbracket \sigma \rrbracket_{\xi}^{K H}$ $=\llbracket \tau \rrbracket_{\xi}^{K H} \Rightarrow \forall \xi . \llbracket \sigma_{\psi} \rrbracket_{\xi}^{K H^{\prime}}=\llbracket \tau_{\psi} \rrbracket_{\xi}^{K H^{\prime}}$ from definition 7.2 is satisfied by lemma 4.5.2(2).

(c) The functor $L: \mathbf{B M M} \rightarrow \lambda \mathbf{2}-\mathbf{M O D}_{\mathrm{s}}$ on objects. Suppose $\mathbf{m} \in \mathbf{B M M}$ with corresponding language $\lambda 2(\mathrm{~m})$ is as described in 7.1 ; a small $\lambda 2$-model $L \mathrm{~m}$ will be built from it step-bystep. The construction is the same as for the term model in 6.1, except that we now work with types and terms from $\lambda 2(\mathbf{m})$ interpreted in $\mathbf{m}$. The syntactical conditions from 7.1.2 force us to proceed like this, in order to ensure well-definedness. The fact that $\lambda 2(\mathbf{m})$ contains type and term constants (cf. 7.1.1) ensures that a (small) $\lambda 2$-model is obtained.

(1) The base category $\mathbf{B}$ of $L \mathrm{~m}$ has objects $n \in \omega$ and morphisms $u=\left(u_{1}, \ldots, u_{m}\right): n \rightarrow m$ are given by functions $u_{i}: T^{n} \rightarrow T$ definable by types, i.e. there is a context $\Gamma=\left\{\gamma_{1}: *, \ldots, \gamma_{n}: *\right\}$ together with types $\sigma_{1}, \ldots, \sigma_{m}$ such that for $1 \leqslant i \leqslant m$ one has $\Gamma \vdash \sigma_{i}: *$ in $\lambda 2(\mathrm{~m})$ and $u_{i}(\mathbf{a})=\llbracket \sigma_{i} \rrbracket_{\xi(\gamma ;=\mathbf{a})}$.

The product of $n$ and $m$ in $\mathbf{B}$ is $n+m$ and 0 is terminal, $\Omega=1 \in \mathbf{B}$; all products $1+\ldots+1 \in \mathbf{B}$ have enough points because $\lambda 2(\mathbf{m})$ has names for all $a \in T$.

(2) A functor $H: \mathbf{B}^{\text {op }} \rightarrow$ Cat is obtained as follows. $H n$ is the category with

obj. $\quad \bar{u}=\lambda \mathbf{a} \in T^{n} . D_{u(\mathbf{a})} \quad$ for $u: n \rightarrow 1 \quad$ in $\mathbf{B}$.

mor. $f: \bar{u} \rightarrow \bar{v}$ are families $\left\{f_{\mathbf{a}}\right\}_{\mathbf{a} \in T^{n}}$ of functions $f_{\mathbf{a}} \in\left[D_{u(\mathbf{a})} \rightarrow D_{v(\mathbf{a})}\right] \quad$ definable by terms:

there must be a context $\Gamma=\left\{\gamma_{1}: *, \ldots, \gamma_{n}: *\right\}$ with types $\sigma, \tau$ defining $u, v$ and a term $M(x)$ such that $\Gamma+x: \sigma \vdash M: \tau$ and $f_{\mathbf{a}}(z)=\llbracket M \rrbracket_{\xi(\gamma:=\mathbf{a})+\rho(x:=z)}$.

For arrows $u: m \rightarrow n$ in $\mathbf{B}$ a functor $u^{*}: H n \rightarrow H m$ is defined by $u^{*}(\bar{v})=\overline{v \circ u}$ and $u^{*}\left(\left\{f_{\mathbf{a}}\right\}_{\mathbf{a} \in T^{n}}\right)=\left\{f_{u(\mathbf{b})}\right\}_{\mathbf{b} \in T^{m}}$. Using the type constants from $\lambda 2(\mathbf{m})$, one sees that $H$ preserves enough points of $1 \in \mathbf{B}$.

(3) The categories $H n$ have a semi-ccc structure as in 6.1. The semi-terminal object is given by $\lambda \mathbf{a} \in T^{n} . D_{\llbracket s t]}: n \rightarrow 1$ in $\mathbf{B}$. The semi-product is $\bar{u} \times \bar{v}=\lambda \mathbf{a} \in T^{n} . D_{u(\mathbf{a}) \& v(\mathbf{a})}$, where \& is the interpretation in $\mathbf{m}$ of the product from 6.1, i.e. $c \& d=\Delta(\lambda b \in T .(c \backsim d \backsim b) \leadsto b)$, with 
ensuing pairing $\left\langle\left\langle_{-},-\right\rangle\right\rangle$and projections $p \in\left[D_{c \& d} \rightarrow D_{c}\right]$ and $p^{\prime} \in\left[D_{c \& d} \rightarrow D_{d}\right]$. The semiexponent is $\bar{v}^{\bar{u}}=\lambda \mathbf{a} \in T^{n} . D_{u(\mathbf{a}) \rightsquigarrow v(\mathbf{a})}$ with

$$
e v=\left\{\lambda z \in D_{(f(\mathbf{a}) \rightsquigarrow g(\mathbf{a})) \& f(\mathbf{a})} \cdot p(z) \cdot p^{\prime}(z)\right\}_{\mathbf{a} \in T^{n}}
$$

and for $f: \bar{w} \times \bar{u} \rightarrow \bar{v}$ one has

$$
\Lambda(f)=\left\{\lambda z \in D_{w(\mathbf{a})} \cdot \lambda y: u(\mathbf{a}) \cdot f_{\mathbf{a}}(\langle\langle z, y\rangle\rangle)\right\}_{\mathbf{a} \in T^{n}} .
$$

We verify that $H 0$ is a $\lambda 1$-model (see definitions 3.3(1) and 2.3.3(2)). First we notice that in $H 0,1_{a, b}=\lambda \mathbf{c} \in T^{0} \lambda z \in D_{\llbracket s t]} \cdot \lambda x: a \leadsto b \lambda y: a \cdot x \cdot y$, where we identify objects of $H 0$ with elements of $T$. Hence if arrows $f, g: t_{0} \rightarrow b^{a}$ satisfy $\forall x: t_{0} \rightarrow a \cdot f \cdot x=g \cdot x$, then for $\mathbf{c} \in T^{0}$ and $z \in D_{[s t]}$ one has $\forall d \in D_{a} . F_{a b}\left(f_{\mathbf{c}}(z)\right)(d)=F_{a b}\left(g_{\mathbf{c}}(z)\right)(d)$, because $\lambda 2(\mathbf{m})$ contains names for $d \in D_{a}$. Hence $1_{a, b} \cdot f=1_{a, b} \cdot g$ and thus we have the Meyer-Scott axiom holds in $H 0$; this makes it a $\lambda 1$-model.

(4) The semi-functors $\Pi_{n}: H(n+1) \rightarrow H n$ are given as follows.

$$
\begin{aligned}
\Pi_{n}(\bar{u}) & =\overline{\hat{u}}, \quad \text { where } \hat{u}(\mathbf{a})=\Delta(\lambda b \in T \cdot u(\mathbf{a}, b)) \\
\Pi_{n}(f: \bar{u} \rightarrow \bar{v}) & =\left\{\lambda z \in D_{\hat{u}(\mathbf{a})} . \lambda b: T \cdot f_{\mathbf{a}, b}(z \cdot b)\right\}_{\mathbf{a} \in T^{n}} .
\end{aligned}
$$

We check that $\Pi_{n}$ is a semi-functor.

$$
\begin{aligned}
\Pi_{n}(g \circ f)_{\mathbf{a}}(z) & =\lambda b: T \cdot g_{\mathbf{a}, b}\left(f_{\mathbf{a}, b}(z \cdot b)\right) \\
& \left.=\lambda b: T \cdot g_{\mathbf{a}, b}\left(\Pi_{n}(f)_{\mathbf{a}}(z) \cdot b\right)\right) \\
& =\Pi_{n}(g)_{\mathbf{a}}\left(\Pi_{n}(f)_{\mathbf{a}}(z)\right) \\
& =\left(\Pi_{n}(g) \circ \Pi_{n}(f)\right)_{\mathbf{a}}(z) .
\end{aligned}
$$

Under the condition that $\mathbf{m}$ satisfies $G_{h} \circ F_{h}=$ id for all $h \in[T \rightarrow T]$, this $\Pi_{n}$ becomes a functor.

$$
\begin{aligned}
\Pi_{n}\left(\mathrm{id}_{\bar{u}}\right)_{\mathbf{a}} & =\lambda z \in D_{\hat{u}(\mathbf{a})} \cdot \lambda b: T \cdot z \cdot b \\
& =\lambda z \in D_{\hat{u}(\mathbf{a})} \cdot\left(G_{u(\mathbf{a},-)} \circ F_{u(\mathbf{a},-)}\right)(z) \\
& =\lambda z \in D_{\hat{u}(\mathbf{a})} \cdot z \\
& =\left(\operatorname{id}_{\Pi_{n}(\bar{u})}\right)_{\mathbf{a}} .
\end{aligned}
$$

The maps $H(n+1)\left(\pi_{n, 1}^{*}(\bar{u}), \bar{v}\left(\rightleftarrows H(n)\left(\bar{u}, \Pi_{n}(\bar{v})\right)\right.\right.$ are defined by

$$
\begin{aligned}
f & \left.\mapsto \lambda z \in D_{u(\mathbf{a})} \cdot \lambda b: T \cdot f_{\mathbf{a}, b}(z)\right\}_{\mathbf{a} \in T^{n}} \\
\left\{\lambda z \in D_{u(\mathbf{a})} \cdot g_{\mathbf{a}}(z) \cdot b\right\}_{\mathbf{a}, b \in T^{n+1}} & \leftrightarrow g .
\end{aligned}
$$

(d) The functor $L: \mathbf{B M M} \rightarrow \lambda 2-\mathbf{M O D}_{\mathrm{s}}$ on arrows. Suppose $\langle\psi,\{\chi\}\rangle: \mathbf{m} \rightarrow \mathbf{m}^{\prime}$ in $\mathbf{B M M}$ as in definition 7.2; we define $L(\langle\psi,\{\chi\}\rangle)=\langle R, S\rangle$ with $R: \mathbf{B} \rightarrow \mathbf{B}^{\prime}$ given by

$$
\begin{gathered}
n \mapsto n \\
u=\left(u_{1}, \ldots, u_{m}\right): n \rightarrow m \mapsto\left(R u_{1}, \ldots, R u_{m}\right),
\end{gathered}
$$

where $R u_{i}$ on $u_{i}=\lambda \mathbf{a} \in T^{n} \cdot \llbracket \sigma_{i} \rrbracket_{\xi_{(\gamma:=\mathbf{a}}}^{\mathbf{m}}$ is defined by $R u_{i}=\lambda \mathbf{a} \in T^{\prime n} \cdot \llbracket\left(\sigma_{i}\right)_{\psi} \rrbracket_{\xi(\gamma:=\mathbf{a})}^{\mathbf{m}^{\prime}}$. This functor 
$R$ is well-defined because the type equalities in $\mathbf{m}$ are also satisfied in $\mathbf{m}^{\prime}$, see definition 7.2. The component-functors $S_{n}: H n \rightarrow H^{\prime} n$ are defined by

$$
\begin{aligned}
\bar{u} & \mapsto \overline{R u} \\
\left\{\lambda z \in D_{u(\mathbf{a})} . \llbracket M \rrbracket_{\xi(y:=\mathbf{a})+\rho(x:=z)}^{\mathbf{m}}\right\}_{\mathbf{a} \in T^{n}} & \mapsto\left\{\lambda z \in D_{R u(\mathbf{a})}^{\prime} \cdot \llbracket M_{\psi, \chi} \rrbracket_{\xi(\gamma:=\mathbf{a})+\rho(x:=z)}^{\mathbf{m}^{\prime}}\right\}_{\mathbf{a} \in T^{\prime}} .
\end{aligned}
$$

(e) An isomorphism $\langle\psi,\{\chi\}\rangle: \mathbf{m} \rightarrow K L \mathbf{m}$ is given by

$$
\begin{aligned}
& \psi(a)=\lambda \mathbf{c} \in T^{0} \cdot a \\
& \chi(d)=\left\{\lambda z \in D_{\llbracket s t \rrbracket} . d\right\}_{\mathbf{c} \in T^{0}},
\end{aligned}
$$

i.e. elements become constant functions and families. By induction one can prove that

$$
\left.\psi \llbracket \sigma \rrbracket_{\xi}^{\mathrm{m}}\right)=\llbracket \sigma_{\psi} \rrbracket_{\psi \circ \xi}^{K L \mathrm{~m}} \quad \text { and } \quad \chi\left(\llbracket M \rrbracket_{\xi+\rho}^{\mathrm{m}}\right)=\llbracket M_{\psi, \chi} \rrbracket_{\psi \circ \xi+\chi \circ \rho}^{K L \mathrm{~m}} .
$$

Obviously, $\langle\psi,\{\chi\}\rangle$ is an isomorphism, which is natural in $\mathbf{m}$. This completes the proof of theorem 7.3.

7.4 .

One might expect to find an arrow $L K H \rightarrow H$ in $\lambda \mathbf{2}-\mathbf{M O D}_{\mathrm{s}}$, but in general one does not know whether the defined semi-terminals and semi-products in fibres of $L K H$ coincide with the given ones in $H$. This is due to the fact that semi-left and right adjoints need not be unique (up to natural isomorphisms) as for ordinary adjoints. Hence in this way the small 22-models $L K H$ and $H$ are incomparable. For the same reason, we cannot say that the term models from 6.1 and 6.2 are initial objects. However, by extending the notion of a BMMmodel with a terminal and with product type representatives - and changing the syntax accordingly - a bit more can be done. For technical reasons (to obtain an adjunction in theorem 7.4.3) we need a normal terminal object; to require only that would be rather ad hoc and so we shall turn to $\lambda \eta 2$-models.

\subsection{1.}

Let $\lambda \eta 2-\times-t$ be the calculus $\lambda \eta 2$ extended with the following rules.

$$
\begin{aligned}
& \vdash \tau_{0}: * \\
& \vdash\langle\rangle: \tau_{0} \\
& \Gamma \vdash \sigma, \tau: * \Rightarrow \Gamma \vdash \sigma \times \tau: * \\
& \Gamma+\Theta \vdash M: \sigma, \Gamma+\Theta \vdash N: \tau \Rightarrow \Gamma+\Theta \vdash\langle\langle M, N\rangle\rangle: \sigma \times \tau \\
& \Gamma+\Theta \vdash Q: \sigma \times \tau \Rightarrow \Gamma+\Theta \vdash p Q: \sigma, \quad \Gamma+\Theta \vdash p^{\prime} Q: \tau .
\end{aligned}
$$

with the conversions $M=\langle\rangle$ for $M: \tau_{0}, p\langle\langle M, N\rangle\rangle=M, p^{\prime}\langle\langle M, N\rangle\rangle=N$ and $\langle\langle p Q$, $\left.p^{\prime} Q\right\rangle>Q$.

\subsection{2.}

Let $\mathbf{B M M} \eta$ be the full subcategory of $\mathbf{B M M}$ determined by $\mathbf{B M M}$-models $\mathbf{m}=\langle T, .$. which satisfy the following requirements. 
(a) There is a type representative $a_{0} \in T$ such that $D_{a_{0}}$ is a singleton (i.e. terminal in Sets), say with $D_{a_{0}}=\{\langle\rangle\}$.

(b) There is a function $\times: T \times T \rightarrow T$, together with pairings $\left\langle\left\langle_{-},-\right\rangle\right\rangle$and projections $p, p^{\prime}$, such that for $a, b \in T$ and $d \in D_{a}, d^{\prime} \in D_{b}$ and $e \in D_{a \times b}$ one has $\left\langle\left\langle d, d^{\prime}\right\rangle\right\rangle \in D_{a \times b}$ satisfying $p\left\langle\left\langle d, d^{\prime}\right\rangle\right\rangle=d, p^{\prime}\langle\langle d, d\rangle\rangle=d^{\prime}$ and $\left\langle\left\langle p e, p^{\prime} e\right\rangle\right\rangle=e$.

(c) $G_{a b} \circ F_{a b}=$ id and $G_{f} \circ F_{f}=$ id.

It is now obvious how to interpret the calculus $\lambda \eta 2-\times-t$ in a model $\mathbf{m} \in \mathbf{B M M} \eta$. (Above we determined $\mathbf{B M M} \eta$ as a full subcategory, thereby implicitly supposing that the syntactical conditions in definition 7.2 already apply to the calculus $\lambda \eta 2-\times-t$.)

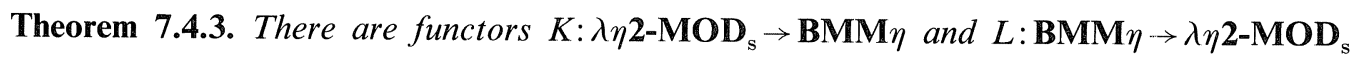
such that $L \dashv K$ with an isomorphism as unit. As a consequence (cf. MacLane, 1971, IV, 3) one has that $L$ is full and faithful.

Proof. The functor $K: \lambda \eta \mathbf{2}-\mathbf{M O D}_{\mathrm{s}} \rightarrow \mathbf{B M M} \eta$ is as defined in 7.3, with the addition of the following two points.

$$
a_{0}=\phi_{t}^{-1}\left(t^{\prime}\right), \quad \text { where } t^{\prime} \in H t \text { is semi-terminal. }
$$

Then one takes \langle\rangle$=\mathrm{id}_{t^{\prime}}: t^{\prime} \rightarrow t^{\prime}$ in $D_{a_{0}}$.

$$
a \times b=\phi_{t}^{-1}\left(\phi_{t}(a) \times \phi_{t}(b)\right)
$$

with $\langle\langle d, e\rangle\rangle=\langle d, e\rangle$ and $p=\lambda z . \pi \circ z, p^{\prime}=\lambda z . \pi^{\prime} \circ z$.

The functor $L: \mathbf{B M M} \eta \rightarrow \lambda \eta \mathbf{2 - M O D}$ is as defined in 7.3, except that for the terminals and products in the fibres we now use those given with the $\mathrm{BMM} \eta$-model instead of the definable canonical ones.

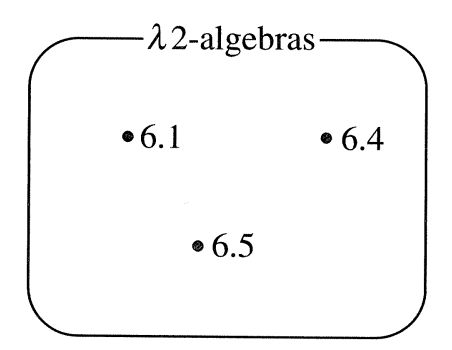

U

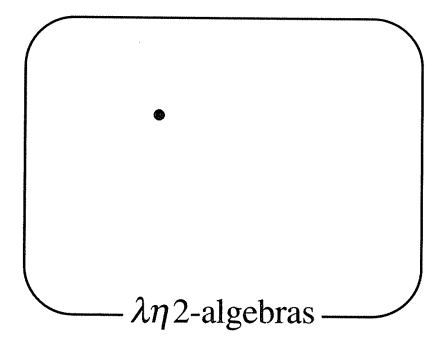

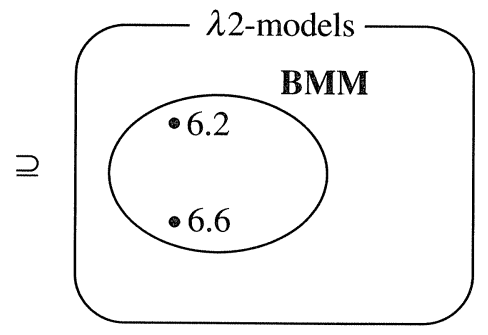

IU

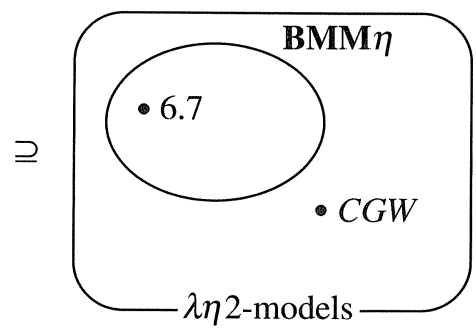

Figure 1 
A unit isomorphism $\mathbf{m} \cong K L \mathbf{m}$ is described in the proof of theorem 7.3 point (e). Furthermore, a counit arrow $\langle R, S\rangle: L K H \rightarrow H$ is layed down by the functor $R: \mathbb{B}^{\prime} \rightarrow \mathbb{B}$ with

$$
\begin{gathered}
n \mapsto \Omega^{n} \\
u=\left(u_{1}, \ldots, u_{m}\right): n \rightarrow m \mapsto\left\langle\llbracket \sigma_{1} \rrbracket_{\gamma: *}^{\mathbf{H}}, \ldots, \llbracket \sigma_{m} \rrbracket_{\gamma: *}^{\mathbf{H}}\right\rangle \circ w_{n},
\end{gathered}
$$

where $\left(\sigma_{1}, \ldots, \sigma_{m}\right)$ define $u$ and $w_{n}=\left\langle!, \pi_{0}^{n-1}, \ldots, \pi_{n-1}^{n-1}\right\rangle: \Omega^{n} \rightarrow t \times \Omega \times \ldots \times \Omega$. By lemma 4.5.2(1), this is well-defined. Notice that there are no problems with constants. The component-functors $S_{n}: H^{\prime} n \rightarrow H \Omega^{n}$ are defined by

$$
\begin{aligned}
& \bar{u} \mapsto \phi_{\Omega^{n}}(R u) \\
&\left\{\lambda z \in D_{u(\mathbf{a})} \cdot \llbracket M \prod_{\xi(y:=\mathbf{a})+\rho(x:=z)}^{K H}\right\}_{\mathbf{a} \in T^{n}} \mapsto w_{n}^{*}\left(\llbracket M \rrbracket_{\gamma: *+x: \sigma}^{H} \circ\langle!, \mathrm{id}\rangle\right) .
\end{aligned}
$$

It takes a bit of puzzling to show that the requirements of definition 3.1.2 are satisfied and that we actually have an adjunction $L \dashv K$.

Remark 7.5. Much of this paper can be summarised in Figure 1.

The numbers refer to the examples from the previous section: 6.1 and 6.2 to term models 6.4, 6.5 and 6.6 to ideal models and 6.7 to the PER-model. The point $C G W$ refers to the domain theoretic model of Coquand et al. (1989). We did not describe an example of a $\lambda \eta 2-$ algebra, but the construction of the open term model of the calculus $\lambda \eta 2-\times-t$ (cf. 7.4.1) as in 6.1 yields one. This will be an initial object (cf. Seely, 1987; 4.5-4.7 or Pitt, 1987, 2.6).

\section{Acknowledgements}

I wish to express my gratitude for helpful discussions with Simone Martini at an early stage of this work and with Henk Barendregt at a late stage. Furthermore, one of the referees provided valuable comments.

\section{References}

Asperti, A. and Longo, G. (1991) Applied Category Theory, book in preparation.

Barendregt, H. P. (1984) The Lambda Calculus. Its Syntax and Semantics (revised second edition), North-Holland, Amsterdam.

Barendregt, H. P. and Rezus, A. (1983) Semantics for classical AUTOMATH and related systems. Inf. \& Contr. 59 127-47.

Bruce, K. B. and Meyer, A. R. (1984) The semantics of second-order lambda calculus. In: G. Kahn, D. B. MacQueen and G. Plotkin, eds, Proc. Conf. on Semantics of Data Types, Sophia-Antipolis, June 1984, Springer Lecture Notes in Computer Science 173 131-144.

Bruce, K. B., Meyer, A. R. and Mitchell, J. C. (1990) The semantics of second-order lambda calculus. Inf. \& Comp. 85 76-134.

Coquand, Th. and Ehrhard, Th. (1987) An equational presentation of higher order logic. In: Pitt et al. (1987) 40-56.

Coquand, Th., Gunter, C. and Winskel, G. (1989) Domain theoretic models of polymorphism. Inf. \& Comp. 81 123-67. 
Ehrhard, Th. (1988) A categorical semantics of constructions. In: Proc. Third Ann. Symp. on Logic in Computer Science, Edinburgh, July 1988, 264-73.

Friedman, H. (1975) Equality between functionals. In: Parikh, R., ed., Logic Colloquium, Symposium on Logic held at Boston, 1972-1973, Springer Lecture Notes in Mathematics 453.

Girard, J.-Y. (1972) Interprération fonctionelle et élimination des coupures de l'arithmétique dordre supérieur. Thèse d'État, Paris VII.

- (1986) The system F of variable types, fifteen years later. Th. Comp. Sci. 22 159-92.

Girard, J.-Y., Lafont, Y. and Taylor, P. (1989) Proofs and types. Camb. Univ. Press Tracts in Th. Comp. Sci. 7.

Goldblatt, R. (1984) Topoi. The categorial Analysis of Logic (revised second edition), North-Holland, Amsterdam.

Hayashi, S. (1985) Adjunction of semifunctors: categorical structures in non-extensional lambdacalculus, Th. Comp. Sci. 41 95-104.

Hoofman, R. (1990) The linear decomposition of $\lambda 2$-models, manuscript, Dep. Comp. Sc. Utrecht Univ., The Netherlands.

Hyland, J. M. E. (1989) A small complete category. Ann. Pure Appl. Log. 40 135-65.

Jacobs, B. (1989) On the semantics of the second order $\lambda$-calculus: from Bruce-Meyer-Mitchell models to hyperdoctrine models and vice-versa. In: D. H. Pitt et a.., eds, Category Theory and Computer Science, Springer Lecture Notes in Computer Science 389 198-212.

Jacobs, B., Margaria, I. and Zacchi, M. (1989) Filter models with polymorphic Types.

Koymans, K. (1982) Models of the lambda calculus. Inf. \& Contr. 52 306-32.

Longo, G. and Moggi, E. (1988) Constructive natural deduction and its 'modest' interpretation. Report CS-88-131, Carnegie Mellon Univ. Also in: MSCS, no. 2, vol. 1.

MacLane, S. (1972) Categories for the Working Mathematician, Springer.

MacQueen, D., Sethi, R. and Plotkin, G. (1986) An ideal model for recursive polymorphis types. Inf. \& Contr. 71 95-130.

Martini, S. (1987) An interval model for second order lambda calculus. In : Pitt et al. (1987) 219-37.

(1988) Modelli non estensionali del polimorfismo in programmazione funzionale. PhD thesis, University of Pisa.

Mitchell, J. C. (1988) Polymorphic type inference and containment. Inf. \& Comp. 76(2/3), 211-49.

Pitt, A. M. (1987) Polymorphism is set theoretic, constructively. In: Pitt et al. (1987) 12-39.

Pitt, D. H., Poigné, A. and Rydeheard, D., eds. (1987) Category Theory and Computer Science, Springer Lecture Notes in Computer Science 283.

Prawitz, D. (1965) Natural Deduction, Almqvist \& Wiksell, Stockholm.

Reynolds, J. (1974) Towards a theory of type structure. In: Paris Colloquium on Programming, Springer Lecture Notes in Computer Science 19 408--25.

Scott, D. S. (1976) Data types as lattices. SIAM J. Comp. 5 522-87.

Seely, R. A. G. (1983) Hyperdoctrines, natural deduction and the Beck condition. Zeitschr. Math. Log. Grundl. Math. 29 33-48.

(1987). Categorical semantics for higher order polymorphic lambda calculus. J. Symb. Log. 52 969-89.

Streicher, Th. (1988) Correctness and completeness of a categorical semantics of the calculus of constructions. Thesis, University of Passau. 